\title{
Turbulence and noise suppression of a high-speed jet by water injection
}

\author{
By A. KROTHAPALLI, L. VENKATAKRISHNAN, \\ L. LOURENCO, B. GRESKA AND R. ELAVARASAN \\ Department of Mechanical Engineering, 2525 Pottsdamer Street, Florida A\&M University \\ and Florida State University, Tallahassee, FL 32310, USA \\ kroth@fmrl.fsu.edu
}

(Received 6 March 2002 and in final form 22 April 2003)

An experimental investigation has been carried out on a supersonic jet of air issuing from an $M=1.44$ converging-diverging rectangular nozzle of aspect ratio 4 . Particle image velocimetry measurements of the flow field along with near-field acoustic measurements were made. The effect of injection of a small amount of water $(\sim 5 \%$ of the mass flow rate of the jet) into the shear layer of the jet, on the unsteady flow structure and sound generation were examined. The presence of water droplets in the jet modified the turbulence structure significantly, resulting in axial and normal r.m.s. velocity reductions of about $10 \%$ and $30 \%$, respectively, as compared to that of a normal jet. An even larger effect is found on the peak values of the turbulent shear stress with a reduction of up to $40 \%$. The near-field noise levels (OASPL) were found to reduce by about $2-6 \mathrm{~dB}$ depending on the location of the injection and the water mass flow rate. Far-field acoustic measurements carried out on a heated $M=0.9$ (jet exit velocity $\left.=525 \mathrm{~m} \mathrm{~s}^{-1}\right)$ jet show significant $(6 \mathrm{~dB})$ reductions in the OASPL with moderate amounts of water injection (17\% of the mass flow rate of the jet) suggesting that the technique is viable at realistic engine operating conditions.

\section{Introduction}

In need of a rational approach to supersonic jet noise suppression, fundamental studies are being conducted in our laboratory using modern experimental techniques. Recognizing that an essential prerequisite is that the nature and location of the important noise sources be clearly understood, our work is focused on describing the unsteady flow of a supersonic jet in some detail using particle image velocimetry (PIV). In this paper, we describe the flow characteristics of an $M=1.44$ ideally expanded rectangular cold jet. It is then followed by a PIV study of the role of water injection on the turbulence characteristics. Concomitantly, near-field acoustic measurements are made to demonstrate the efficacy of this technique for noise suppression. Encouraged by the near-field noise measurements, an additional experiment is conducted to obtain the far-field noise measurements in a recently built high-temperature anechoic jet facility using a heated $M=0.9$ axisymmetric jet, with and without water injection. The exit velocity of the axisymmetric jet is comparable with that of the rectangular jet.

The far-field mixing noise of a supersonic jet appears to be comprised of three major noise components (Crighton 1977). The first is a field coherent in phase, but of high frequency and short wavelength. The flow-visualization pictures show that the waves, commonly referred to as Mach waves, have plane phase fronts and are 
confined to a definite wedge sector. They emanate from the region within the first few diameters downstream of the nozzle exit. These are generated by small-scale disturbances (or eddies) that are being convected at supersonic speeds so that they emit Mach waves in the direction defined by a disturbance convection velocity and the atmospheric speed of sound (Phillips 1960; Ffowcs Williams 1965; Ffowcs Williams \& Maidanik 1965). Although these waves lie in an important range of the spectrum ( $1 \sim 4 \mathrm{kHz}$ in the case of full scale engines), they may not have enough intensity at far distances to contribute significantly to the far-field noise. These waves are eliminated by surrounding the jet with a gas stream that has a higher speed of sound, thus resulting in subsonic convection velocities of the small disturbances, as demonstrated by Oertel \& Patz (1981) and more recently by Papamoschou (1997).

The second field is highly directional, peaking at smaller angles relative to the jet axis (or larger angles to the inlet axis). This noise field is generated from large-scale instabilities reaching peak amplitude in the region somewhat upstream of the end of the potential core. These sources are associated with the unsteady flow on a scale that is comparable with the local shear layer width (Bishop, Ffowcs Williams \& Smith 1971). The spectral intensity of this sound field generally contains two distinct peaks (Laufer, Schlinker \& Kaplan 1976). One is associated with highly directional Mach waves characterized by high positive pressure peaks in the far-field microphone signal (Ffowcs Williams, Simson \& Virchis 1975; Laufer et al. 1976). These Mach waves are of significant strength as compared to those seen very close to the jet exit as discussed above. It is found that the far-field intensity contribution of this source is about $30 \%$ of the measured total intensity (Laufer et al. 1976; Krothapalli, Venkatakrishnan \& Lourenco 2000b). This intense radiation is observed to emanate from a region between 5 and 10 nozzle diameters and is associated with supersonically travelling largescale coherent regions of vorticity (Oertel, Gatau \& George 1981). The sources for the second peak appear to be located much farther downstream $(10 \sim 20$ nozzle diameters). These sources are associated with the unsteady flow generated by the large structures similar to that in subsonic jets.

The third noise component is directionally independent and consists of higher frequencies. This sound is generated in precisely the same manner as in subsonic flow by the fine-scale chaotic turbulence. Analysis of experimental data by Tam (1998) vividly shows the contributions of the distinct components to the total far-field spectrum: the Mach wave radiation due to large-scale motions and radiation due to small-scale turbulence.

This paper presents a possible approach for the suppression of the dominant largescale mixing noise sources in a supersonic jet. Water droplets are injected into the jet to manipulate the dominant source region, which extends typically from 5 to 20 diameters from the nozzle exit. The dispersed phase serves to attenuate the turbulent kinetic energies of the gas phase in the noise-producing region of the jet. The strength of the attenuation is expected to depend upon the mass of water injected (mass loading), the droplet size and the injection location. The contribution to the noise by supersonically convecting eddies is not significant in this experiment owing to the relatively low convective Mach number of the jet. The convective Mach number $\left(M_{c}=\left(U_{e}+a_{e}\right) /\left(a_{\infty}+a_{e}\right)\right.$, where $U_{e}$ and $a_{e}$ are the jet exit velocity and speed of sound, respectively, and $a_{\infty}$ is the ambient speed of sound, Oertel 1979) of the cold rectangular jet is about 1.16 whereas it is 1.19 for the heated axisymmetric jet. The Mach wave radiation becomes significant only for $M_{c}>1.25$ (Krothapalli, Arakeri $\&$ Greska 2003) and as such the contributions to the far-field noise, in the present experiments, are largely due to the mixing noise. 
Sound attenuation due to the presence of water droplets in air has been a subject of study since 1948 (Knudsen, Wilson \& Anderson 1948). Marble \& Wooten (1970) and Marble \& Candel (1975) conducted theoretical investigations to determine the sound attenuation in ducts by vaporization of liquid droplets. For plane waves, the attenuation magnitude exceeds $5 \mathrm{~dB} \mathrm{~m}^{-1}$ at a temperature of $25^{\circ} \mathrm{C}$ with a cloud of $0.7 \mu \mathrm{m}$ radius droplets constituting $1 \%$ of the gas mass (Marble \& Candel 1975).

Recent experimental studies of rocket exhaust noise suppression by Zoppellari \& Juve (1997) show that injecting a large amount of water (several times the mass of the jet) into a supersonic jet results in a significant decrease $(\sim 10 \mathrm{~dB})$ in the far-field noise. However, this procedure may not be practical for aircraft jet engine application. Hence, in the present investigation we limit the amount of water injected to a small fraction of the total mass of the jet $\left(\dot{m}_{\text {water }} / \dot{m}_{\text {air }} \approx 0.1\right)$.

Because of the difficulty in measuring velocity fields in supersonic flows, few attempts have been made in the past to characterize supersonic jets with the degree of thoroughness found in subsonic jets. Using laser-Doppler velocimetry (LDV), Lau, Morris \& Fisher (1979) obtained a limited amount of data on an $M=1.37$ axisymmetric jet. A discussion relating to this data is deferred to later in the paper. Non-axisymmetric jets, such as rectangular jets have noise attenuation properties in the plane that is prone to flapping oscillation. This is clearly demonstrated by Hoch \& Hawkins (1974) using an axisymmetric notched nozzle and more recently by Alvi et al. (1996) and Seiner et al. (1992) using non-axisymmetric nozzles. This asymmetry of the flow provides a significant opportunity in the control of high-speed jet noise. Keeping this in mind, as a first step in a systematic study, the present experiment is designed to investigate the effect of water injection on the turbulence characteristics in the noise-producing region of a rectangular supersonic jet. To further validate the concept of water injection on noise suppression, far-field noise results of experiments on a heated axisymmetric jet operating at an exit Mach number of 0.9 are also included in the paper.

\section{Apparatus, instrumentation and procedures}

\subsection{Experimental facilities}

Experiments were conducted in two different blow-down facilities of the Fluid Mechanics Research Laboratory. The facility used for the rectangular-jet experiments is located in a non-anechoic room and is used for the PIV and near-field sound measurements. The round-jet study was conducted in an anechoic hot-jet facility to obtain far-field noise measurements. The high-pressure air for both facilities is supplied from a bank of storage tanks with a total capacity of $10 \mathrm{~m}^{3}$. A high-displacement reciprocating air compressor, which is capable of supplying air at a maximum storage pressure of 160 bars, drives the facilities.

\subsubsection{Non-anechoic jet facility}

In the non-anechoic jet facility, the air can be heated by passing through an array of resistive tank heaters having a maximum power output of $450 \mathrm{~kW}$ and capable of achieving stagnation temperatures up to $700 \mathrm{~K}$. The $M=1.44$ jet used in this study can be run continuously for about $40 \mathrm{~min}$ at constant temperature. The facility was fitted with a rectangular nozzle having an exit aspect ratio of $4: 1$. The dimensions of the nozzle in the exit plane were height, $h=10 \mathrm{~mm}$, by width, $w=40 \mathrm{~mm}$. The contour of the short dimension of the nozzle was generated using the method of characteristics for a design Mach number of 1.44. The walls of the long dimension 
of the nozzle were parallel downstream of the throat. The circular dimension of the connecting pipe (diameter $=70 \mathrm{~mm}$ ) upstream of the throat was blended to facilitate a smooth transition to a rectangular cross-section. During all experiments, the ratio of the stagnation to ambient pressure was held nominally at 3.37, as required for isentropically expanded flow at Mach 1.44. The jet was operated at a stagnation temperature of $335 \mathrm{~K}$ to avoid problems of condensation associated with the humid Florida air. The stagnation pressure and temperature were held constant to within $0.5 \%$ of its nominal value during the experiment.

The mean exit velocity profile with laminar boundary layers was top-hat. The jet was exhausted into a quiescent surrounding at ambient conditions. The centreline mean exit velocity, $U_{e}$, is measured to be about $444 \mathrm{~m} \mathrm{~s}^{-1}$, corresponding to a Reynolds number based on the nozzle height of about $4.3 \times 10^{5}$. The convective Mach number of the initial shear layer $M_{c}=1.16$. Significant Mach wave radiation is generally seen when $M_{c}$ exceeds about 1.25 . For this reason, Mach wave radiation is not expected to be a significant component of the far-field radiated noise.

For PIV studies, the jet was seeded with small $(\sim 0.5 \mu \mathrm{m})$ oil droplets generated by a liquid atomizer seeder, the details of which can be found in Alkislar (2001). The seeder unit is placed into a tank that can be pressurized up to $1 \mathrm{MPa}$. The tank also serves as a reservoir for the seeding liquid. Seeded air from the atomizer is mixed with the main air supply stream in the pipe at $1.25 \mathrm{~m}$ upstream of the nozzle. The ambient air was seeded with smoke particles $(1 \sim 5 \mu \mathrm{m}$ in diameter) produced by a Rosco fog generator. Larger particles in the ambient air that are entrained into jet are expected to be confined to mostly subsonic regions of the flow field. Recent measurements, obtained in an underexpanded jet created using the same apparatus, showed that the velocity measurements obtained using PIV are in close agreement with those derived from total and static pressure measurements (Alkislar 2001). Because the particles were exposed to relatively weak compression and expansion of the flow within the shock cells, very little particle lag was noticed in the measurements. In the present experiment, the nozzle being operated at the ideally expanded condition, no shock cells were observed. The velocity of the particles was found to be in close agreement $( \pm 1 \%)$ with the calculated exit velocity using isentropic relations.

A Cartesian coordinate system $(x, y, z)$ was chosen with its origin located at the centre of the nozzle exit plane and with the $x$-axis oriented along the centreline of the jet, $y$ - and $z$-axes are oriented along the short and long dimensions, respectively (see figure 6). The measurements are confined to the central plane $((x, y)$-plane) of the jet containing the small dimension of the nozzle and, $(y, z)$-planes at selected locations downstream of the nozzle exit.

\subsubsection{Anechoic hot-jet facility}

A brief description of a new facility that was used to generate the heated axisymmetric jet is given in this section. The supply air is heated by passing through a sudden expansion (SUE) burner that uses ethylene as the fuel. The burner has an inlet diameter of $76.2 \mathrm{~mm}$ and an exit diameter of $152.4 \mathrm{~mm}$ with an overall length of $0.813 \mathrm{~m}$ and it is cooled by a water jacket. To minimize the effect of any combustion instabilities on the flow, a $1.525 \mathrm{~m}$ long water-cooled pipe having a diameter of $152.4 \mathrm{~mm}$ is used. Following the water-cooled extension are three sections: a measurement section; an extension section; and a reduction section. Each of them is made of a nickel-based alloy, Nistele 230, which can withstand temperatures in excess of $1400 \mathrm{~K}$ without the need for cooling. The conditions of the flow before reaching the nozzle are carefully monitored and managed within these sections. 
Immediately following the water-cooled extension is the measurement section. This section is $0.61 \mathrm{~m}$ long and has an inside diameter of $152.4 \mathrm{~mm}$. The first $50 \mathrm{~mm}$ of this section contains a ceramic honeycomb followed by two titanium screens to suppress any large-scale disturbances in the flow. Midway into the section are four equally spaced ports along the circumference that are $2.54 \mathrm{~mm}$ in diameter. Two of the opposing ports are used to measure the static pressure of the airflow. It turns out that at this location the static and stagnation pressures are nearly equal. The other two opposing ports have Omega C-type thermocouples inserted through them so that they can measure the stagnation temperature of the airflow. The extension section follows the measurement section. This section is also $0.61 \mathrm{~m}$ long and has an inside diameter of $152.4 \mathrm{~mm}$. Immediately after the extension section is the reduction section. It is $0.61 \mathrm{~m}$ long; with its inside diameter varying linearly from $152.4 \mathrm{~mm}$ at its inlet to $76.2 \mathrm{~mm}$ at the exit. A nozzle with a $76.2 \mathrm{~mm}$ inlet diameter can then be attached directly to the exit of the reduction section. In the present experiments, a converging axisymmetric nozzle having an exit diameter of $50.8 \mathrm{~mm}$ was used. The nozzle upstream of the exit was designed using a fifth-order polynomial with a contraction ratio of approximately 2.25 . The stagnation pressure and temperature were held constant to within $0.5 \%$ of its nominal value during the experiment.

The jet exhausts into an anechoic chamber $5.2 \mathrm{~m}$ wide, $5.8 \mathrm{~m}$ long and $4 \mathrm{~m}$ high. The walls, ceiling and floor are covered with high-temperature $305 \mathrm{~mm}$ deep wedges. The wedges are composed of \#703 fibreglass and they have a fibreglass cloth covering followed by a mesh covering. The chamber has provisions for adequate ventilation for easy entrainment of the ambient air into the chamber during jet operation. The exhaust system was also treated to minimize any noise generated by the impinging flow. The cut-off frequency of the chamber is about $300 \mathrm{~Hz}$.

The jet exhausted into a quiet surrounding at ambient conditions. The stagnation temperature of the primary jet was kept at $1033 \mathrm{~K}$. The jet exit velocity for the condition of $M_{j}=0.9$ is $525 \mathrm{~m} \mathrm{~s}^{-1}$. The corresponding Reynolds numbers, based on the nozzle exit diameter is $2.75 \times 10^{5}$. The convective Mach number of the initial shear layer $M_{c}=1.19$ which is nearly the same as that in the cold supersonic jet used in the PIV studies. As in the cold supersonic jet, the Mach wave radiation is not expected to be a significant component of the far-field radiated noise. Because of the restrictions imposed on jet seeding in the anechoic chamber that is necessary for PIV measurements, only far-field acoustic measurements are carried out.

\subsection{Water injection}

The configuration employed for water injection into the shear layer of the rectangular cold jet is shown in figure 1 . The water was atomized and injected into the jet by means of four 1993 Mitsubishi Eclipse fuel injectors (Model MPFI 629-098 Type 1.8L) two for each shear layer. The injectors were pressurized to $1.55 \mathrm{MPa}$, and pulsed at $100 \mathrm{~Hz}$. The injectors were located at 13 heights above and below the jet centreline and at an angle of $16^{\circ}$ to it. The water exited the injectors as a spray cone. In the expectation that the noise-producing region would be located at several heights downstream of the nozzle exit, most of the detailed investigations were carried out with the water injected into the shear layer covering the region from $10 \mathrm{~h}$ to $17 \mathrm{~h}$ (zone I in figure 1; $h$ is the nozzle exit height). Although this region accounts for the bulk of the water entrainment, some of the water was carried along and entrained into the shear layer downstream of this location. This also accounts for the appearance of a few large droplets near the edge of the jet downstream of the injection region. Because of the constraints posed by the PIV image-acquisition optics, the velocity field 


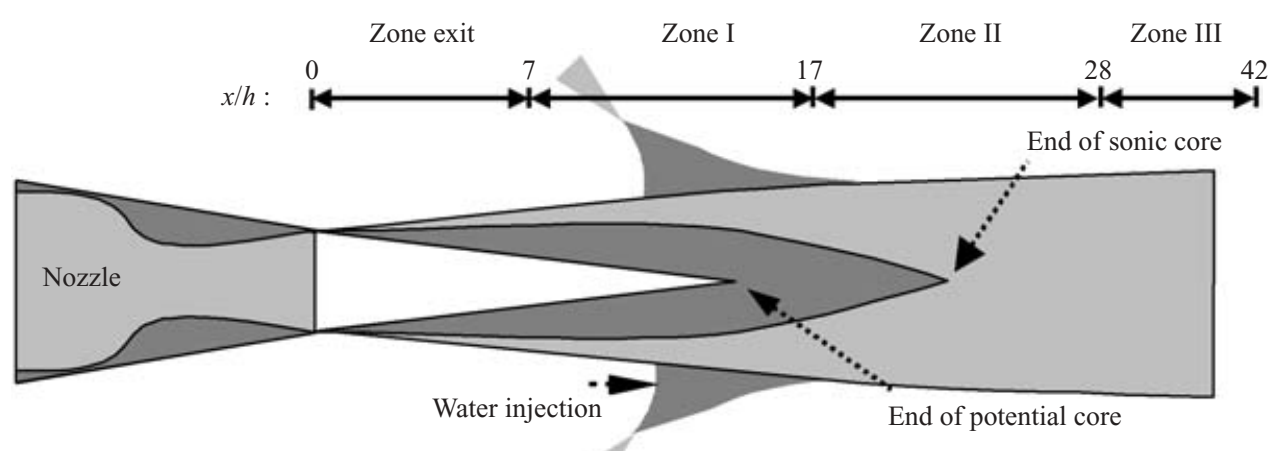

FIGURE 1. Diagram of the water injection set-up.
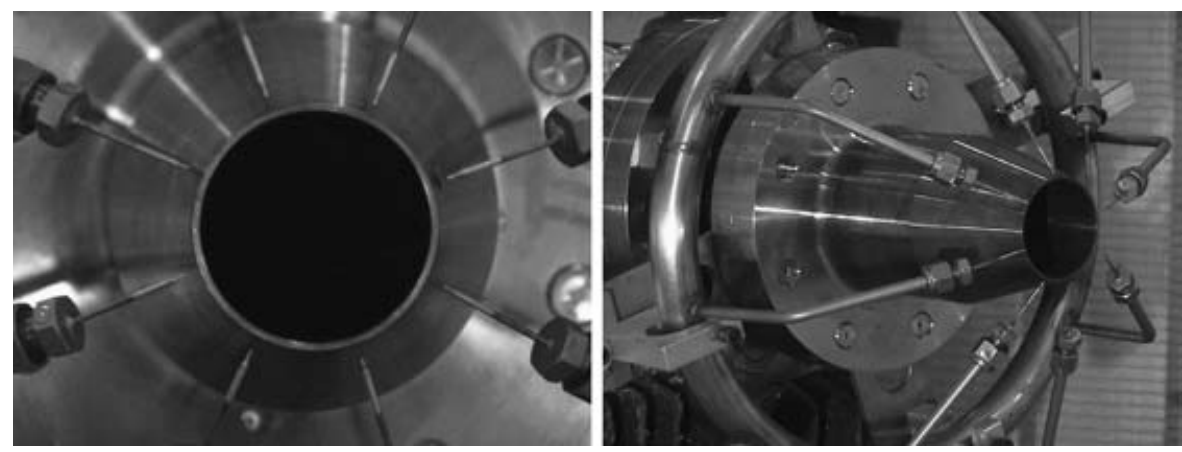

FIGURE 2. The converging axisymmetric nozzle with micro nozzles used for water injection.

data were obtained in different regions of the flow, marked as zone exit to zone III. From the probability density function of droplet diameter at the injector exit, the arithmetic mean diameter $\left(D^{10}\right)$ is found to be $140 \mu \mathrm{m}$. The velocity of the drops at the injector exit was $20 \mathrm{~m} \mathrm{~s}^{-1}$ and decreases to about $10 \mathrm{~m} \mathrm{~s}^{-1}$ at the edge of the shear layer. When exposed to the high shear, the drops break up quickly from an initial size of $140 \mu \mathrm{m}$ to a few micrometres in zone II. The water droplet characteristics when exposed to the high-speed shear layer are discussed in the Appendix. For the PIV experiment, the mass of the water injected was limited to $5 \%$ of the jet mass flow. The resulting volume flow rate is about 1.0 litre $\mathrm{min}^{-1}$. To observe the effect of the water mass flow rate on the near-field acoustics, it was increased to about $10 \%$ of the jet mass flow.

For the heated jet experiments reported here, a converging axisymmetric nozzle with an exit diameter of $50.8 \mathrm{~mm}$ was used. The water is injected though micro-nozzles that were made out of $400 \mu \mathrm{m}$ stainless steel tubing. Keeping in mind the practical application of the technique, the water was injected at the nozzle exit as opposed to far downstream. The water microjets impinge on the shear layer at $6 \mathrm{~mm}$ downstream of the nozzle exit. The angle of the microjets with respect to the upstream jet centreline was $60^{\circ}$. Eight microjets were used, but experiments were also carried out with four and sixteen microjets. The microjet arrangement is shown in figure 2 . The stagnation pressure of the microjets was varied from $2.07 \mathrm{MPa}$ to $3.45 \mathrm{MPa}$ corresponding to a mass flow rate of $12 \%-17 \%$ of the primary jet mass flux. The high pressure helps to create the appropriate spray consisting of droplets. It is expected that they will 
have characteristics similar to those of the fuel injectors used for the PIV study. At lower microjets pressures, the effects were found to be less effective. From simple calculations, it is found that the time scale for the evaporation of the droplets is much larger than the droplet breakup process that is in effect to yield the observed results.

\subsection{Particle image velocimetry}

Non-intrusive measurements of the velocity field were made using PIV. While a stereoscopic PIV system was used to measure the cross-planes of the jet, most of the measurements are confined to the central plane of the jet containing the small dimension of the nozzle using a conventional PIV (one camera placed perpendicular to the plane of interest). A detailed discussion of the application of the stereoscopic PIV technique to supersonic jets is given in Alkislar, Krothapalli \& Lourenco (2003).

The CCD camera used to capture the images had a resolution of $1008(\mathrm{H}) \times 1018(\mathrm{~V})$ pixels with the size of $9 \times 9 \mu \mathrm{m}$, and a maximum framing rate of $30 \mathrm{~Hz}$. The camera was equipped with a $58.37 \mathrm{~mm}$ focal length lens that was specifically designed for the wavelength of the laser light. A microcomputer, with two Pentium II CPUs, controlled the camera, and was capable of acquiring up to 128 image pairs at the maximum camera rate. To illuminate the flow field, a frequency doubled Nd-Yag laser with dual cavity (Spectra-Physics PIV-400) was used. The time $\Delta t$ between the two laser pulses was kept at $1.3 \mu \mathrm{s}$. The camera was positioned at right-angles to the jet axis. The optical arrangement was set such that the image area covered was about $12 \mathrm{~cm} \times 12 \mathrm{~cm}$.

An image-matching approach is used for the digital processing of the image pairs to produce the displacement field. To achieve velocity data with high spatial resolution, a novel-processing algorithm was developed (Lourenco \& Krothapalli 2000). With the new processing approach, the particle images themselves comprise the interrogation region, which have sizes ranging from 3 to 4 pixel squares. In this scheme, the particle images in the correlation window are detected by a threshold method based on the image intensity gradient. Then the background level is removed as well as the images of particles lying on the boundaries of the interrogation window. A correlation is then carried out with the remaining 'masked' image data based on each individual particle. The displacement between image pairs was found in the usual manner by means of cross-correlation, and a velocity (displacement) vector is assigned at the middistance between image pairs. Therefore, each particle pair contributes to a second order approximation of the velocity. However, in contrast to the traditional approach, which uses structured grids, these velocities are evaluated in an unstructured grid. The flow field at any point is described by an analytical function using a least-squares fitting algorithm. The function that is used is a second-order polynomial,

$$
\boldsymbol{u}=\boldsymbol{a} x^{2}+\boldsymbol{b} x+\boldsymbol{c} y^{2}+\boldsymbol{d} y+\boldsymbol{e} x y+\boldsymbol{f} .
$$

The marked advantage of this approach is that the field is described at any point with accuracy of second order, including the derivatives that are found by differentiating the previous equation. The error minimization approach maintains the order of the accuracy and provides a means for accurate evaluation of the field derivatives. It was demonstrated that this approach provides substantial improvement in accuracy and spatial resolution over traditional PIV methods (Lourenco \& Krothapalli 2000).

Although an unstructured grid is used for calculating the velocity, for ease of presentation, the velocity field is usually presented at regular intervals. The time it takes to compute a vector field depends on the computer hardware. Processing speed 
ranges from 1400 interrogations s ${ }^{-1}$ on a $200 \mathrm{MHz}$ dual Pro Pentium PC up to several thousand on a $500 \mathrm{MHz}$ Alpha based PC.

\subsection{Acoustic measurements}

The near-field acoustic measurements of the rectangular jet were made with $1 / 4$ in diameter B\&K type 4136 microphones. The normal protection grid was used to prevent any accidental damage. The microphone was used in conjunction with the B\&K Nexus Model 2960 amplifier. A National Instruments PCI-MIO-16E-1 A/D card, capable of sampling at $1.2 \mathrm{MHz}$, in conjunction with a Pentium II $333 \mathrm{MHz}$ PC was used for data acquisition. The sampling rate was $200 \mathrm{kHz}$. At each location of the microphone, 409600 samples were collected and analysed with a 4096 point fast Fourier transform (FFT) and averaged. The resulting resolution of the narrowband spectra is $48.8 \mathrm{~Hz}$. The sound pressure level is defined in the conventional manner, $\mathrm{SPL}=20 \log _{10}\left(p_{\text {rms }} / p_{\text {ref }}\right)$, where $p_{\text {ref }}$ is taken as $20 \mu \mathrm{Pa}$. The overall sound pressure level (OASPL) was calculated by numerical integration of the spectra.

The spectra were taken at increments of $1^{\circ}$ along a circular arc of radius $0.56 \mathrm{~m}$ $\left(r / D_{e}=25, D_{e}=\right.$ hydraulic diameter $)$. All the data were taken in the central plane containing the minor axis of the nozzle (minor axis plane). The microphone was mounted on a traverse controlled by high-resolution stepper motors. The entire data collection was accomplished using LABVIEW software. No corrections to data are made due to the frequency response of the microphone. The jet issues into a nonanechoic room, as such these measurements are only used to note any changes brought about by water injection as compared to the normal jet.

Far-field acoustic measurements of the hot axisymmetric jet are made in the anechoic room. Acoustic instrumentation consisted of ten Bruel \& Kjaer model 4939 microphones with B\&K model 2670 preconditioning amplifiers and three B\&K model 2690 Nexus conditioning amplifiers. The microphones were set up in an arc that had a radial distance of 64 diameters from the nozzle exit. The arc covered the polar angle, $\theta$ ranging from $90^{\circ}$ to $150^{\circ}$ relative to the jet inlet axis.

Each of the microphones had a relatively flat frequency response up to $100 \mathrm{kHz}$ and was sampled at $250 \mathrm{kHz}$. The dataset for each microphone contained 409600 samples (1.6s). This allowed for an FFT of 4096 points over 100 subsets. Averaging the results for the 100 subsets reduced the random error in the calculation to within $0.1 \%$. The resulting narrowband spectrum had a spectral resolution of $61 \mathrm{~Hz}$.

A number of corrections are applied to the microphone signals to obtain accurate data. The corrections for the actuator response as well as the free-field response are applied at each frequency. Lastly, the effect of atmospheric absorption at each frequency was also determined and applied through the use of formulae provided by Blackstock (2000). The corrected SPL values are then converted back into pressure values and integration is then performed over the corrected spectrum. The resulting squared pressure value can then be used to obtain the OASPL.

\section{Results and discussion}

\subsection{Instantaneous velocity field characteristics}

The detailed investigation of the rectangular jet characteristics was carried out using the PIV technique. Typical double-exposure images of the jet with water injection are shown in figure 3 . The measurements presented in this section are obtained in the central plane containing the small dimension of the nozzle $((x, y)$-plane). The main jet is seeded with oil droplets while the ambient medium is seeded with smoke 


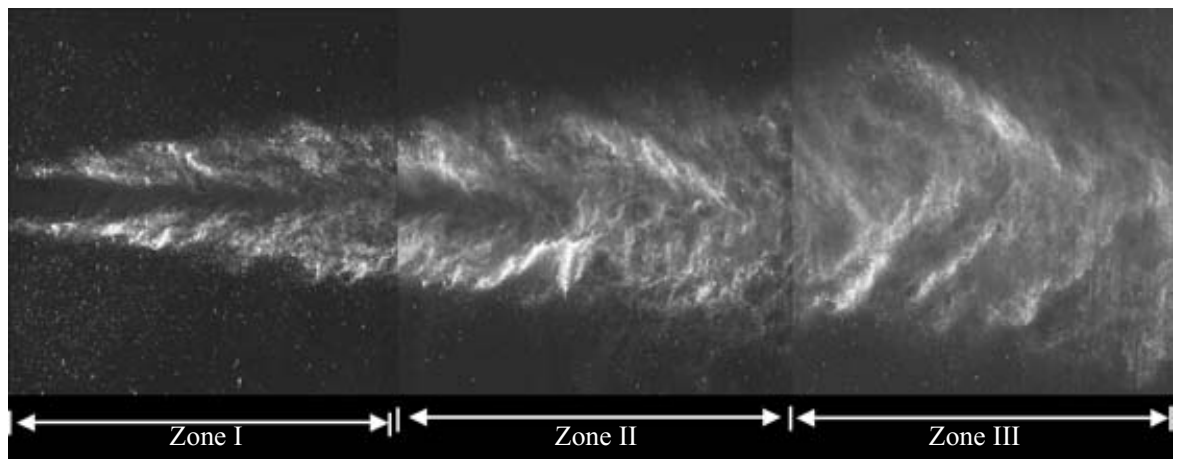

FIGURE 3. Double-exposed PIV images in the central $(x, y)$-plane of the rectangular jet.

particles. Owing to the limitations of the CCD sensor resolution, the images were taken covering each region, as marked in figure 1, separately. For most of the results discussed here, the water is injected in the region denoted in figure 1 as zone I. Because of the intense scattering from water droplets, they can be distinguished easily from the seed particles in the PIV images as shown in figure 3. In zone I, most of the injected water is confined to the shear layer, indicating that the breakup of the large water drops takes place in the shear layer. The measured droplet p.d.f. at the end of this region and beyond, suggests that the breakup process was completed within zone I, and the arithmetic mean droplet diameter at the end of the region was found to be $4 \mu \mathrm{m}$. The discussion related to the water droplet characteristics is given in the Appendix. In zones II and III, it was clearly seen that the water droplets were smaller in size and more uniformly distributed in the jet. Velocity measurements in the injection zone were not obtained because of the difficulties encountered by the CCD sensor saturation and laser beam attenuation.

The instantaneous velocity fields in zones II and III were obtained by the method described in $\S 2.1$ with interrogation regions of $16 \times 16$ pixels corresponding to a physical dimension of $2.3 \mathrm{~mm} \times 2.3 \mathrm{~mm}$. The data were obtained using a $110 \times 80$ $(x, y)$ Cartesian grid. Typical instantaneous velocity fields in zone II corresponding to the normal jet and the jet with water injection are shown in figure 4 . The velocity field is shown as uniformly scaled vectors. One thousand such instantaneous velocity fields were obtained for each of the conditions tested. It is to be noted that the velocity field described here is that of the gas phase (air jet) and most of the water droplets, being of the order of $1 \sim 4 \mu \mathrm{m}$ act like seeding particles.

To accentuate the large-scale vortical structures, the velocity field is plotted in a reference frame moving at a uniform velocity of $100 \mathrm{~m} \mathrm{~s}^{-1}$. When plotted in this fashion, large-scale flapping motion of the natural jet is clearly depicted in figure 4 . The dominant effect of water injection is the reduction of the flapping motion of the jet. In addition, the size of the recognizable large-scale vortical structures is also reduced. From a number of instantaneous pictures in zone II, the convection velocity of the large vortical structures was found to be about $50 \%$ of the local centreline velocity. The suppression of the large-scale motions in the jet with water injection is quite evident in these velocity fields.

A further analysis of the instantaneous velocity fields was conducted to extract the quantitative information related to length scale changes corresponding to the large-scale motions in the jet; this was done using two-point spatial correlations of 

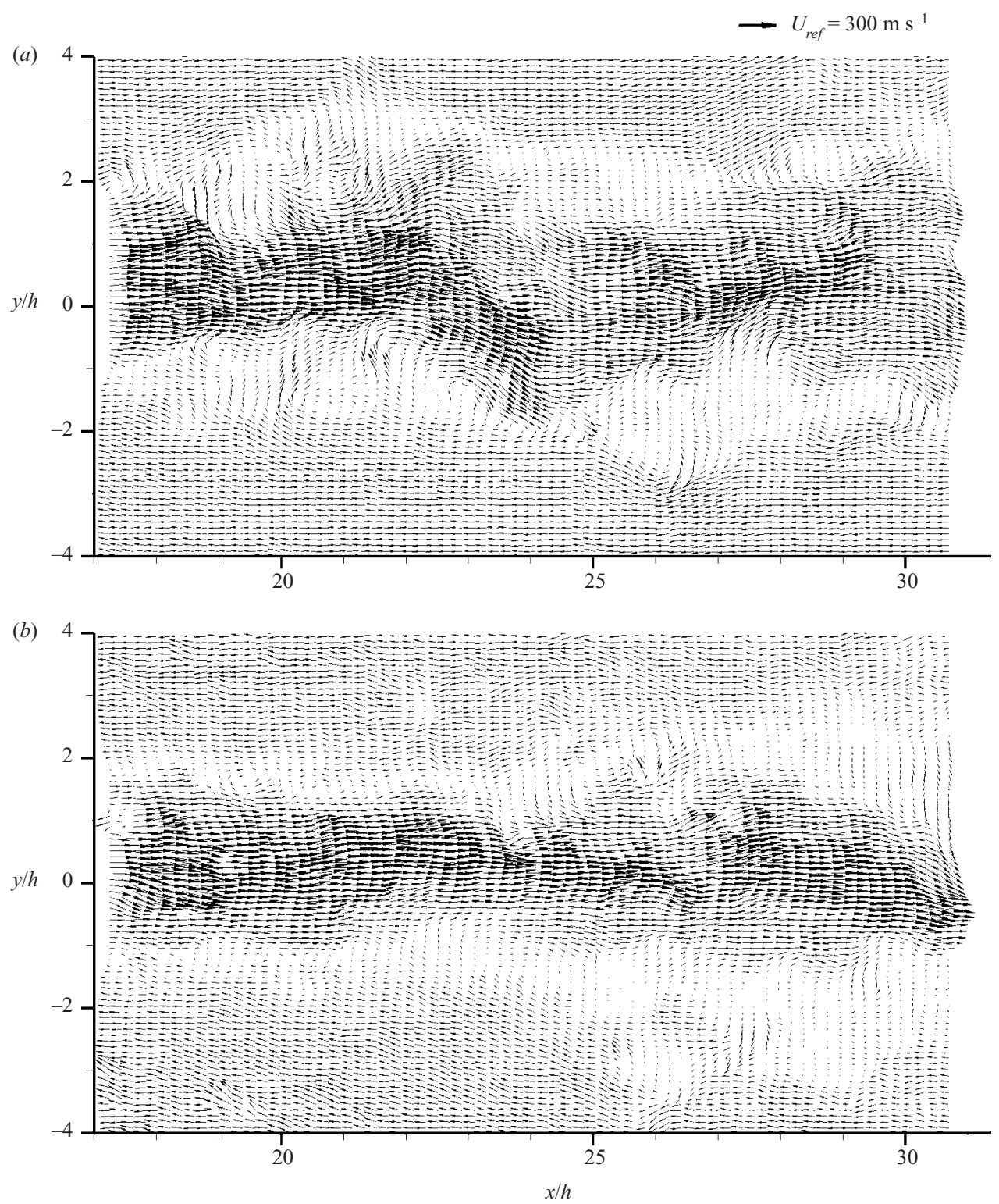

FIGURE 4. Typical instantaneous velocity fields of the rectangular jet in the central $(x, y)$-plane of zone II (reference frame moving with $100 \mathrm{~m} \mathrm{~s}^{-1}$ ). (a) Normal jet; $(b)$ with water injection.

the axial component of the velocity, defined as

$$
R_{u u}=\frac{\left\langle u\left(x_{0}, y_{0}, t\right) u(x, y, t)\right\rangle}{\left\langle u\left(x_{0}, y_{0}, t\right) u\left(x_{0}, y_{0}, t\right)\right\rangle} .
$$

Here, $u$ is the axial component of velocity and $x_{0}$ and $y_{0}$ is the reference location in the flow. The high value of the correlation function $R_{u u}$ indicates the presence of a spatially coherent region. Typical contours of $R_{u u}$ at a specific location in the upper shear layer of the jet in zone II are shown in figure 5. The size of the well-correlated region $\left(R_{u u}>0.4\right)$ can be clearly identified in the figure. A characteristic length 

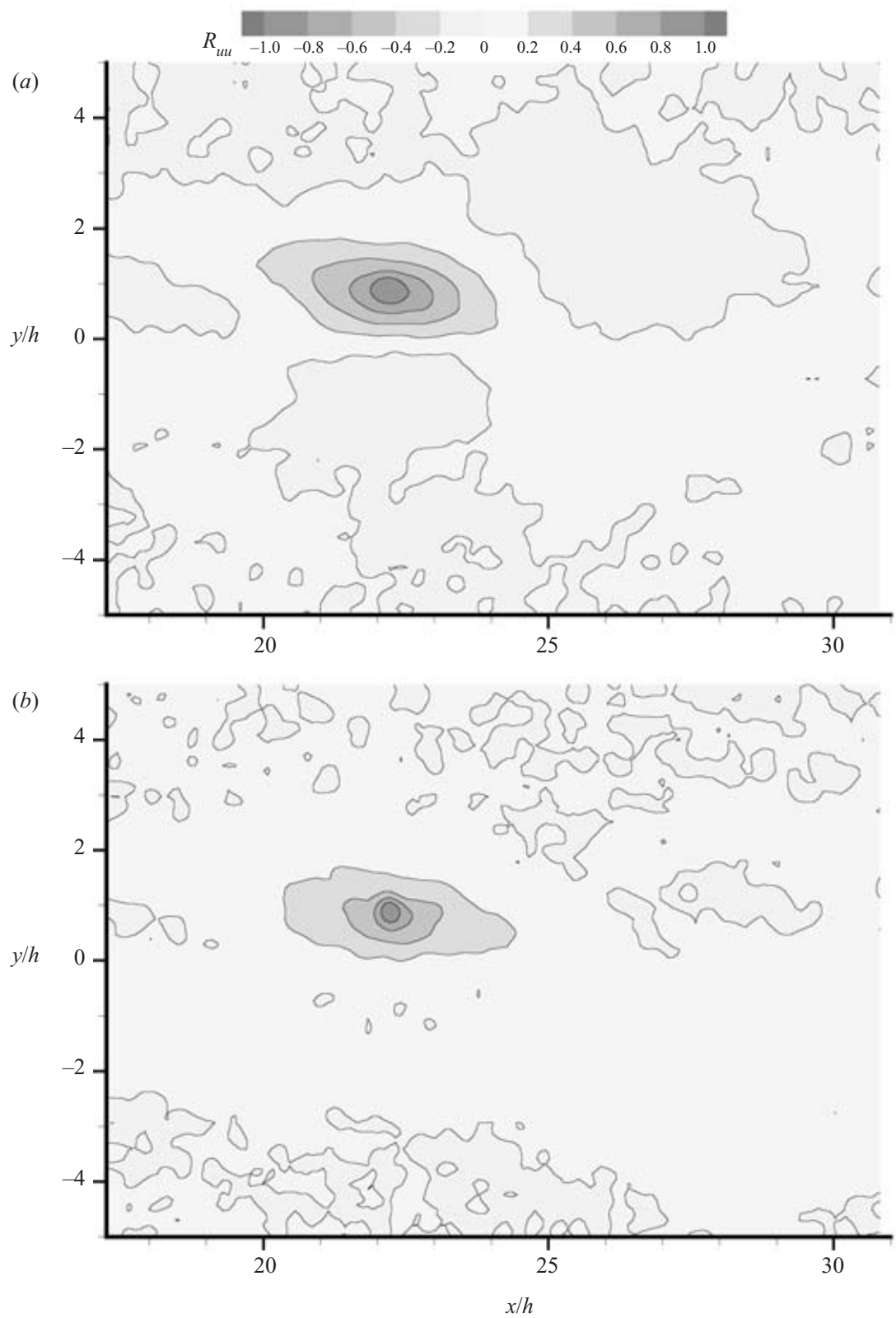

FIGURE 5. Contours of two-point spatial correlation function in the upper shear layer of the rectangular jet (central $(x, y)$-plane) at $x / h=22$. (a) Normal jet; $(b)$ with water injection.

scale is calculated from the well-correlated contour map that may be considered as the measure of the average size of an eddy. The variation of the equivalent length scale obtained at various downstream locations in the region corresponding to the maximum shear shows a monotonic increase of the eddy size with downstream distance similar to that of Lau (1980) (Krothapalli et al. 2000a). The length scale at a downstream location of $8 h$, without water injection, is measured to be about $0.3 h$, which is in agreement with the measurements of Lau (1980) made on an $M=1.37$ axisymmetric jet. The length scale obtained with water injection reduces the 


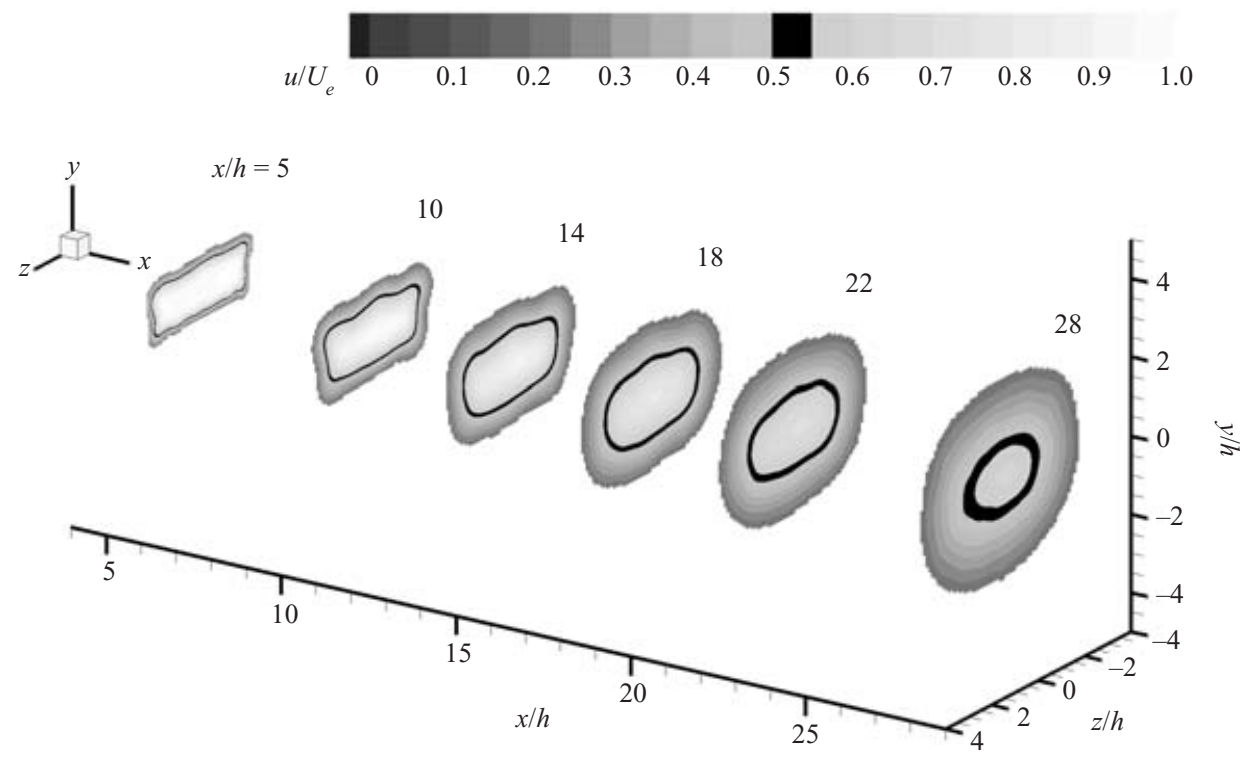

FIGURE 6. Contours of the axial mean velocity at different cross-planes of the rectangular jet.

magnitude of the eddy size at least within the region of the measurement $(x / h<42)$. The effect of the water droplets in the flow is to reduce this macro length scale of the flow by nearly half. This observation is consistent with the reduced size of the vortical structures with water injection observed in the instantaneous velocity field (figure 4). The reductions in the length scales with water injection are observed in the post-injection region up to a downstream distance of about $45 h$.

\subsection{Mean and turbulent velocity characteristics}

Distributions of the mean and r.m.s. fluctuating velocities in the central $(x, y)$-plane of the jet with water injection are presented and compared with those of the reference or normal jet. In order to use and interpret the values of estimated statistical parameters (e.g. mean velocity, r.m.s. velocity) the accuracy of these estimates and the degree of confidence attached to them must be addressed. The number of samples required to estimate the mean value within a certain precision depends on the level of agitation. On the other hand, the relative error in the r.m.s. estimate is only a function of the number of samples used in the estimate. From previous supersonic jet investigations using LDV (Lau et al. 1979), it is known that the r.m.s. velocity reaches a maximum value of about $15 \%$ of the mean. The number of samples used to obtain the statistical quantities, in the present experiment, is 1000 . With $90 \%$ confidence level, the errors in the mean and r.m.s. values are estimated to be about $1 \%$ and $5 \%$, respectively. However, in the far downstream region, the data appear to show significant $(20 \%)$ variations in fluctuating quantities. We speculate that these large variations may be due to camera vibration. Since, the location of the camera with respect to the jet centreline remains fixed, the spreading jet in close proximity gives rise to image shifting.

\subsubsection{The mean flow structure}

The cross-plane mean velocity fields, obtained using the stereoscopic PIV, at different downstream locations, as shown in figure 6, best capture the three-dimensional features of the jet evolution. The velocity distributions are represented by the contours 

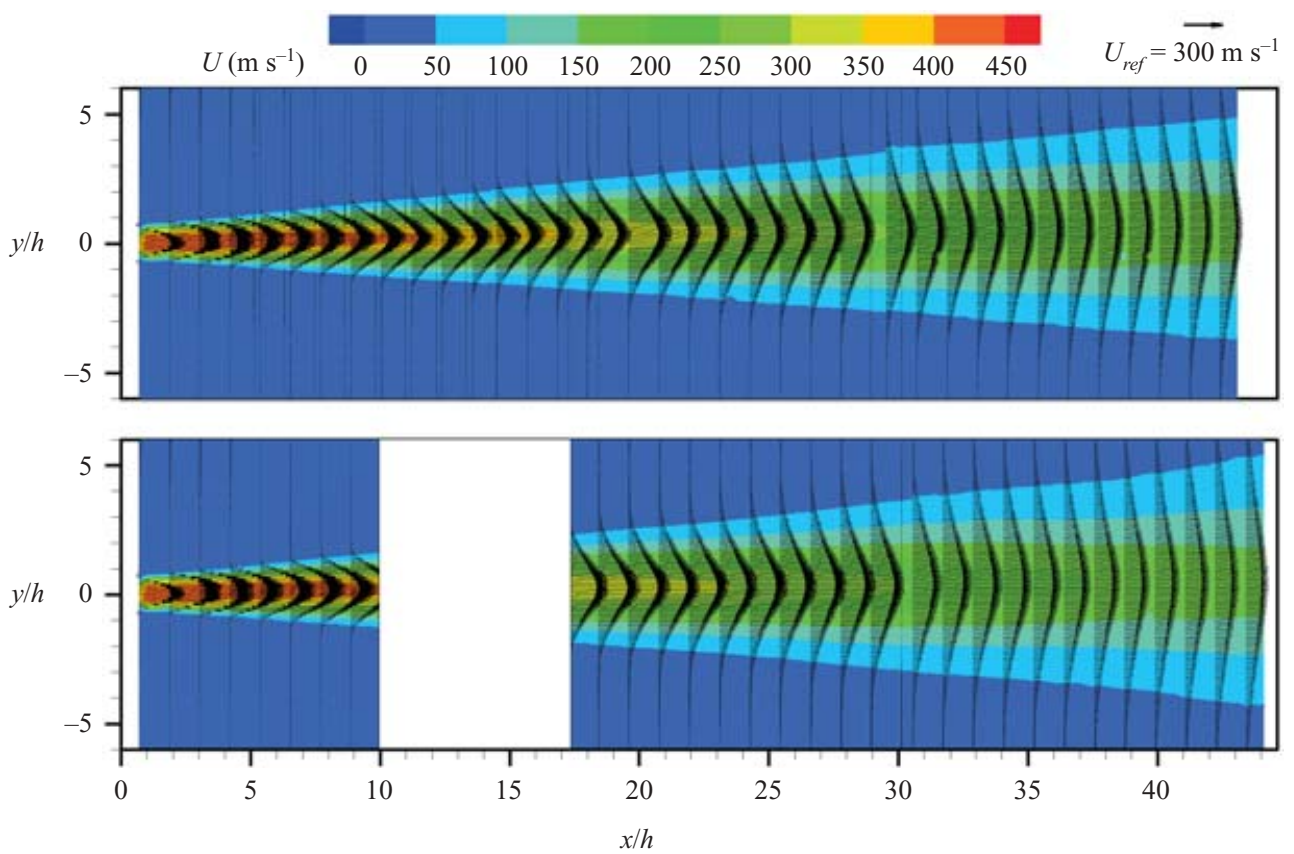

FIGURE 7. Mean axial velocity field in the central $(x, y)$-plane of the rectangular jet. (a) Normal jet; (b) with water injection.

of the axial mean velocity magnitude normalized by the mean jet exit velocity $\left(U_{e}=444 \mathrm{~m} \mathrm{~s}^{-1}\right)$. The axis-switching phenomenon, commonly observed in low-speed jets (Krothapalli, Baganoff \& Karamcheti 1981), may occur at a downstream location beyond the region considered in this study. The jet appears to preserve its rectangular cross-section within the region of interest here. As such, most of the measurements discussed in the rest of the paper are confined to the central $(x, y)$-plane of the jet. A detailed discussion on the cross-over phenomenon and its relevance to supersonic jet development is discussed by Alkislar et al. (2003).

The mean velocity distribution in the central $(x, y)$-plane, covering the region from the nozzle exit to about $42 \mathrm{~h}$ is shown in figure 7. The end of the potential core and the sonic core are found to be at $8 h$ and $22 h$, respectively. The end of the potential core in subsonic jets, is generally identified as the location where large-scale vortical structures in the shear layers merge at the jet centreline.

Except in the immediate neighbourhood of the post-injection region, the velocity fields in the jets with and without water injection are similar, suggesting that the water injection has minimal effect on the mean flow structure, as shown in figure 7. Further evidence of it can be found from the results presented in figure 8 , where the normalized centreline mean axial velocity variation is shown for the two cases. A slight reduction of the velocity magnitude seen in the beginning of zone II is representative of the particle lag associated with relatively larger water droplets present in the injection region. For downstream distances beyond about $25 \mathrm{~h}$, the centreline velocity decays as $x^{-1 / 2}$, at a rate about the same as that of the plane jet. The small variations in the mean velocity with downstream distance seen within the first few heights are due to the presence of weak compression and expansion waves in the jet column.

Figure 9 shows the growth of the jet in the central plane containing the small dimension of the nozzle. The ordinate, $b_{1 / 2}$ is the distance from the centreline of the 


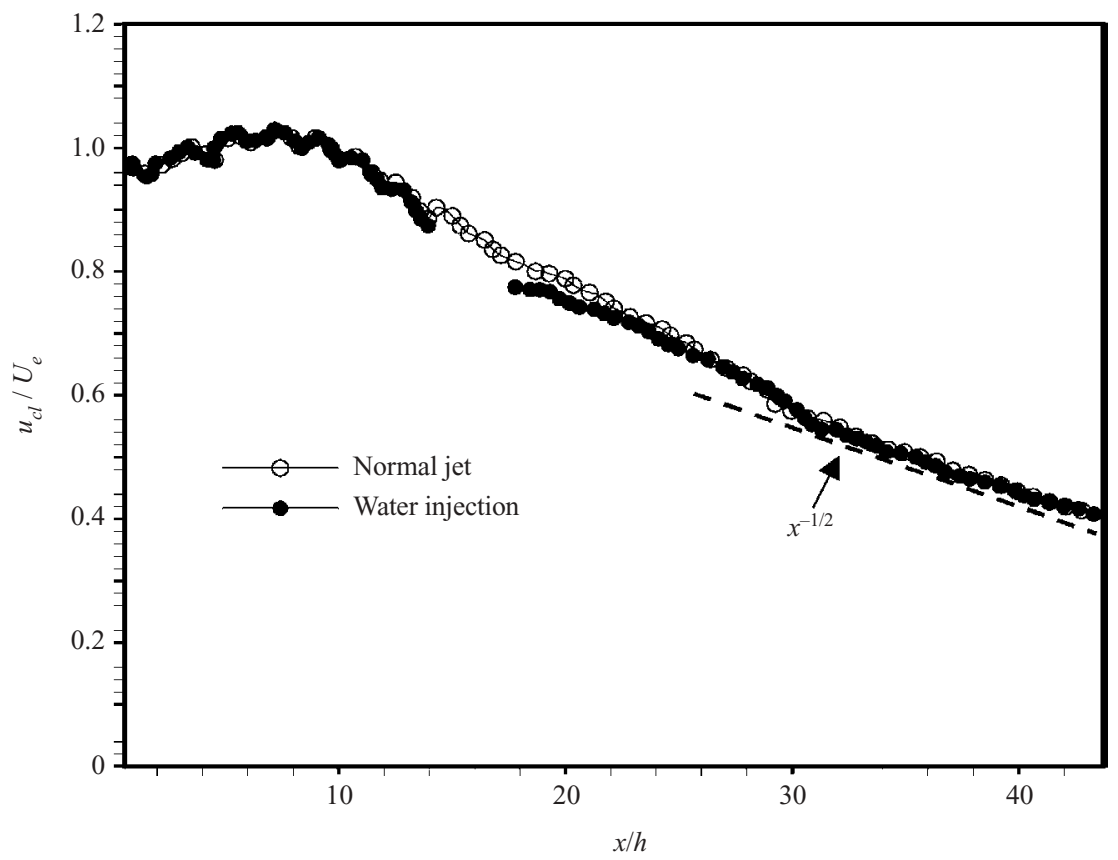

FIGURE 8. Mean axial centreline velocity variation of the rectangular jet with downstream distance.

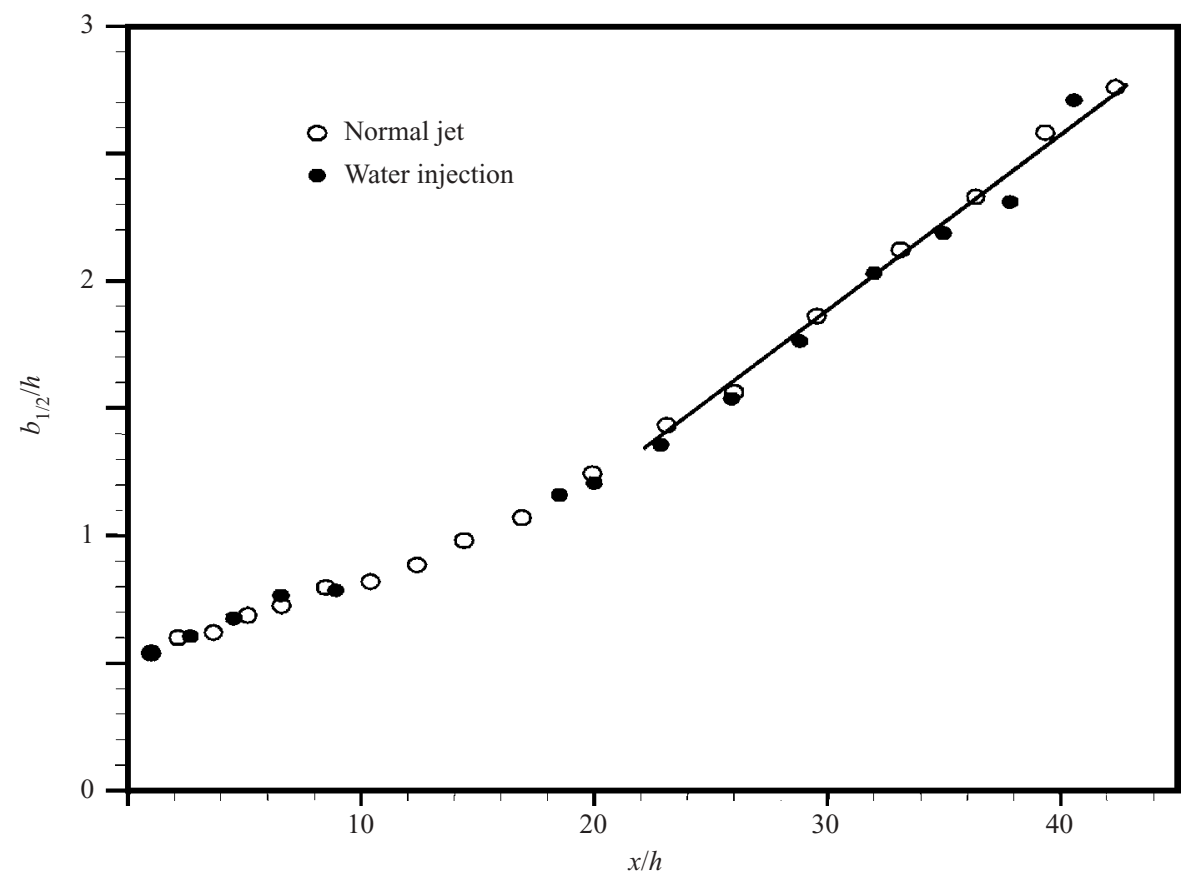

FIGURE 9. Variation of the half velocity width with downstream distance in the central $(x, y)$-plane of the rectangular jet. 
jet to the point where the mean velocity is equal to half its centreline value. The jet spreads linearly with $x$ for $x / h>20$ for both the normal jet and the jet with water injection. The half velocity width of the jet is given by

$$
b_{1 / 2}=k\left(x-x_{0}\right),
$$

where $k=0.07$ and $x_{0}=2 h$. The value of $k$ is in accordance with the previous measurements of Krothapalli et al. (1986). The solid line in the figure is drawn to represent the linear variation.

Figure 10 shows the distribution of the axial mean velocity across the jet at selected downstream locations, ranging from 25 to 40 heights. The velocity $u$ is normalized with respect to the $u_{c l}$, centreline velocity, while the distance $y$ is normalized by the corresponding half-width $b_{1 / 2}$. The profiles are geometrically similar, within the limits of error for the experiment, for $x$ greater than about $25 \mathrm{~h}$. The shape of the profiles is quite similar to that observed by Krothapalli et al. (1986). This similarity persists even in the case of the jet with water injection. These measurements clearly suggest that the mean flow structure of the jet, in the post-injection region, is minimally affected by water injection. Kulick, Fessler \& Eaton (1994) have also found that the presence of solid particles in the boundary layer of a channel flow do not affect the mean profiles while appreciably reducing turbulence intensities as is the case in the present investigation. This observation is attributed to the energy transfer between turbulence scales, a matter still being studied by the two-phase flow workers (Kulick etal. 1994; Mashayek 1998).

\subsubsection{Turbulence characteristics}

Figure 11 shows the centreline distribution of the axial and transverse turbulence intensities $\left(u^{\prime}=u_{r m s} ; v^{\prime}=v_{r m s}\right)$. These values are normalized with respect to the mean axial jet exit velocity. The magnitudes of the turbulence intensities increase close to the jet exit reaching a peak at about $16 h$ and subsequently remain almost unchanged with downstream distance. The peak values of the axial and transverse turbulence intensities are observed to be about 0.125 and 0.085 , respectively. The measured magnitudes are close to those of Lau et al. (1979), made in an $M=1.37$ axisymmetric jet. The location at which these peak amplitudes are observed is also consistent with their measurements. The jagged variation of the r.m.s. intensities with downstream distance, in the downstream region, as mentioned earlier, may be due to camera vibration. The dotted and solid lines shown through the data indicate the best fit. In the case of the jet with water injection, a significant reduction in transverse turbulence intensity is observed in the post-injection region. As shown in figures 12-14, the reductions observed extend into the entire post-injection region of the jet.

The turbulence intensity and shear stress fields in zone II for both the cases of normal jet and the jet with water injection are shown in figures 12-14. The magnitude of the turbulence quantity shown in colour contours is superimposed on the mean velocity field. The magnitudes of the turbulence intensities and the shear stress of the water-injected jet are substantially reduced as compared to those of the normal jet in particular, the maximum shear zone. The maximum reductions are found in the distribution of the transverse turbulence intensity and the shear stress. For example, the maximum intensity of $v$ ' fluctuations is about 0.08 and the contour corresponding to this level covers almost the entire jet column. While in the case of the water-injected jet, the maximum contour level decreases to about 0.055 , almost a $30 \%$ reduction in magnitude. Much greater reductions (about 60\%) are seen in the turbulent shear stress distribution (figure 14). The reductions in the streamwise fluctuations are not as 

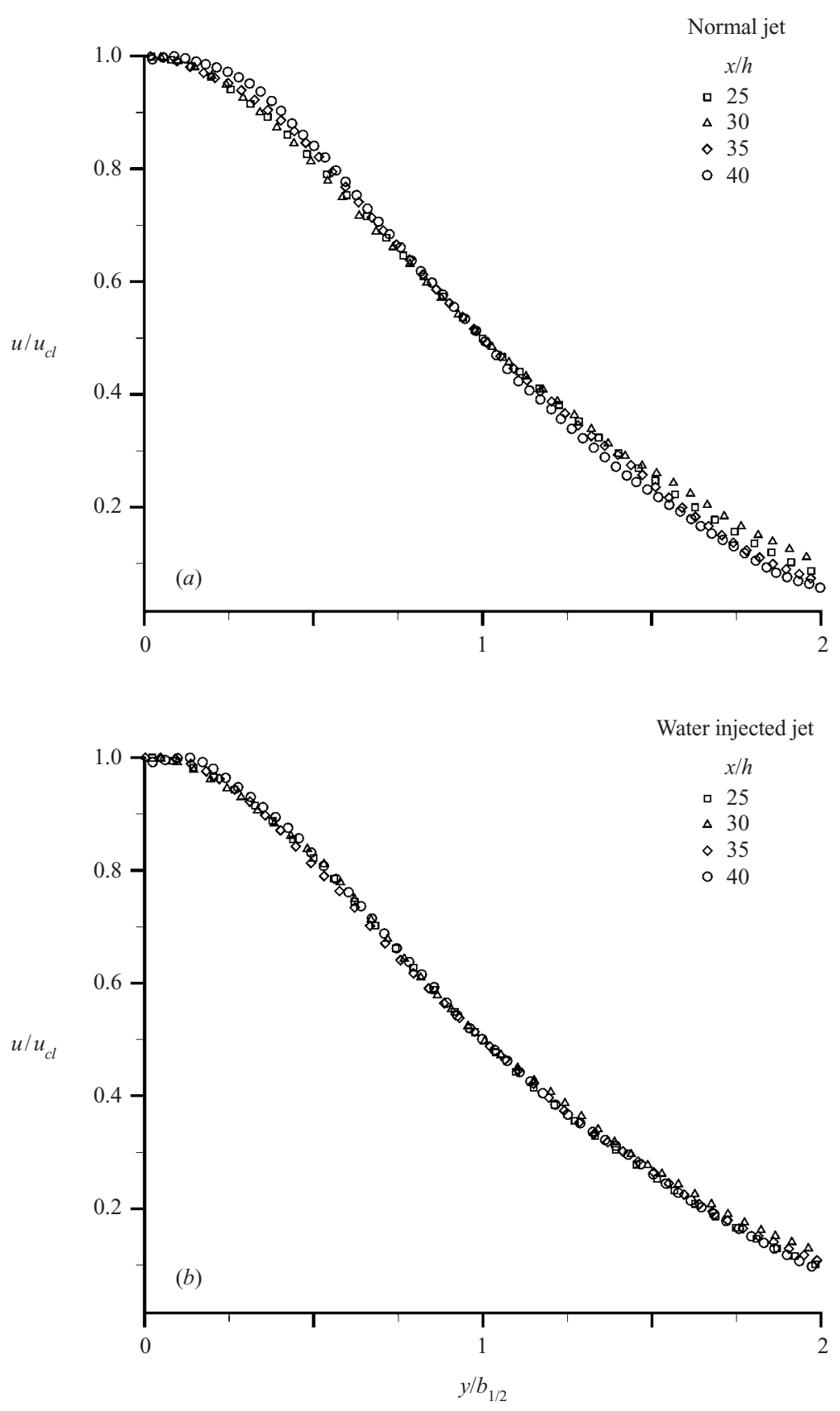

FIGURE 10. Normalized mean axial velocity profiles of the rectangular jet in the post-injection region.

significant, giving rise to increased anisotropy of the flow. The measurements made in the cross-planes using stereoscopic PIV show similar results. The number of samples used to obtain the averages is 300 . As such, the contour edges are not smooth. An example of the transverse velocity fluctuation $\left(v^{\prime}\right)$ field at $x / h=25$ is shown in figure 15. The magnitude of the turbulence quantity shown in colour contours clearly shows the reductions in the turbulence intensity over an extended region of 


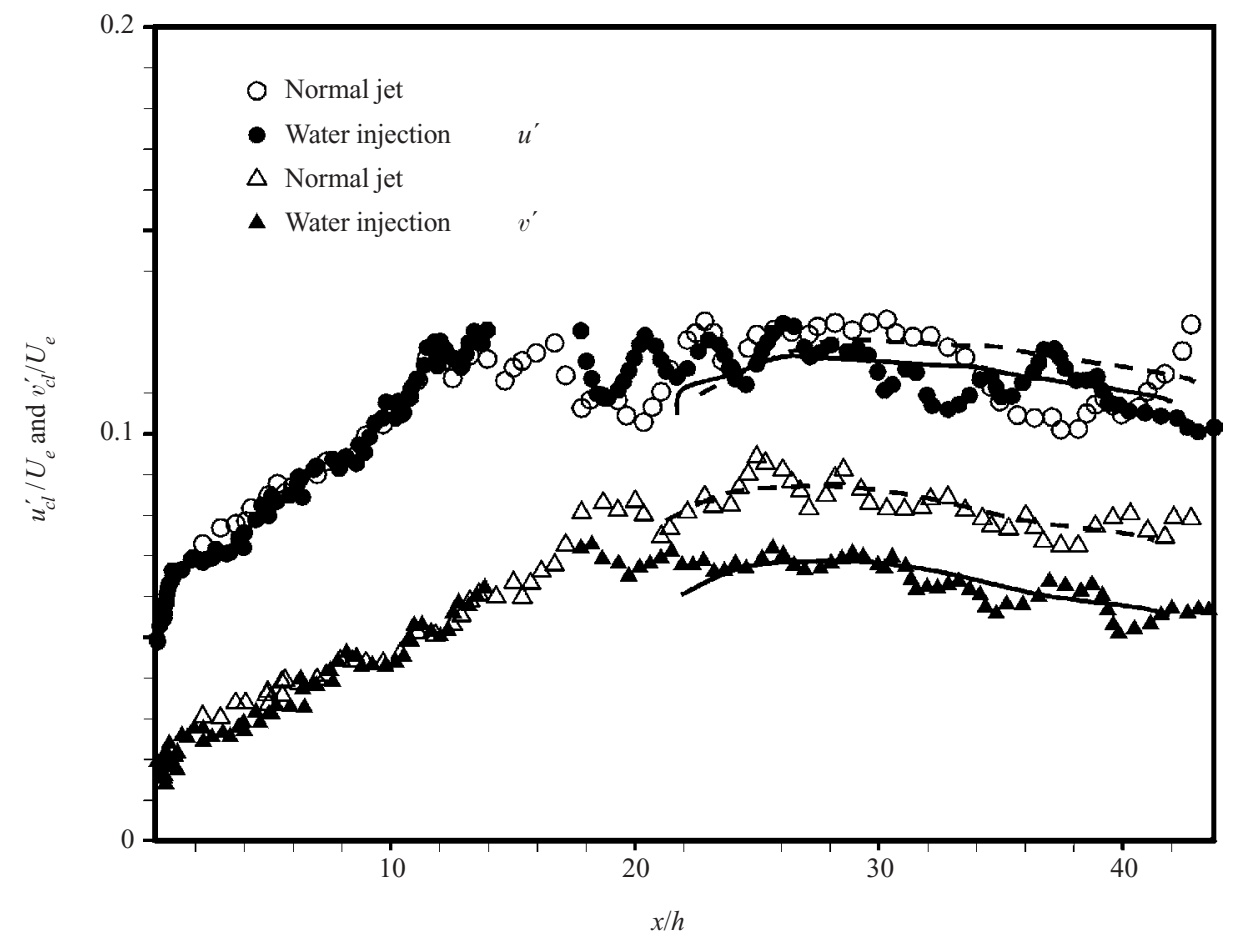

FIGURE 11. Variation of the normalized centreline turbulence intensities with downstream distance of the rectangular jet.

the jet. Also shown in figure 16 are the reductions in the cross-plane turbulent shear stress distribution $v^{\prime} w^{\prime}$, at $x / h=25$. Similar reductions are also seen in zone III, except the magnitudes of the reductions seen are much lower. These measurements clearly indicate that the effect of water injection is to lower the turbulence intensities, (especially in $v^{\prime}$ ) and turbulent shear stress in the jet. Turbulence attenuation in particle laden flows has been observed in shear flows (Kulick et al. 1994), but most often the mass loading is much higher than in the present situation. Recent DNS simulations of droplet-laden flows by Mashayek (1998) show similar trends to those observed in this experiment.

\subsection{Acoustic measurements}

\subsubsection{Rectangular-jet near-field noise measurements}

Acoustic measurements at $r / D_{e}=25\left(D_{e}=\right.$ equivalent diameter $)$ were made to examine the effect of water injection on the sound generation. The measurements were made in a non-anechoic room and as such the measurements are only used for relative comparisons. The noise data were taken at two conditions, which include water injection, using spray nozzles, at the end of the potential core and injection with water jets at the nozzle exit at a maximum mass flow rate of about $10 \%$ of the primary jet mass flow rate. The narrowband spectra shown in figure 17 include the data for the two cases. Although the data were taken at $1^{\circ}$ increments from $90^{\circ}$ to $130^{\circ}$, only a selected set of spectra are presented here. The angle is measured from the jet inlet axis. The near-field spectra show that the water injection at the nozzle exit has the maximum effect and noise suppression also increases dramatically when the mass flow rate is changed from $5 \%$ to $10 \%$. The water injection appears to have an effect 


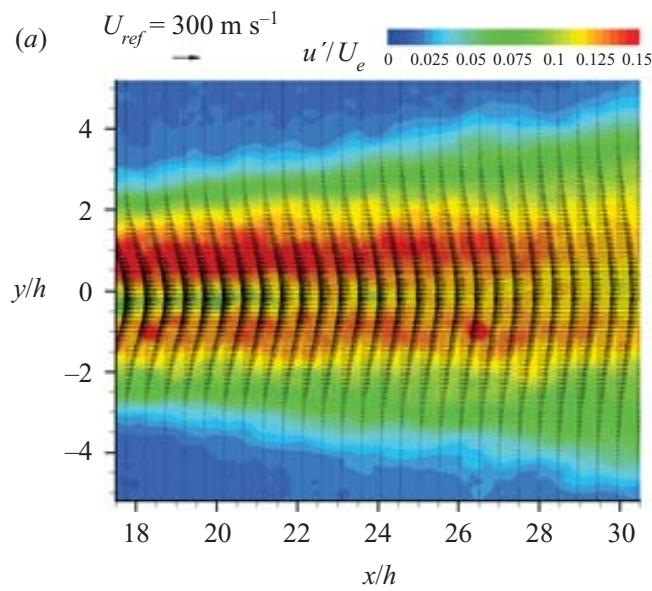

(b)

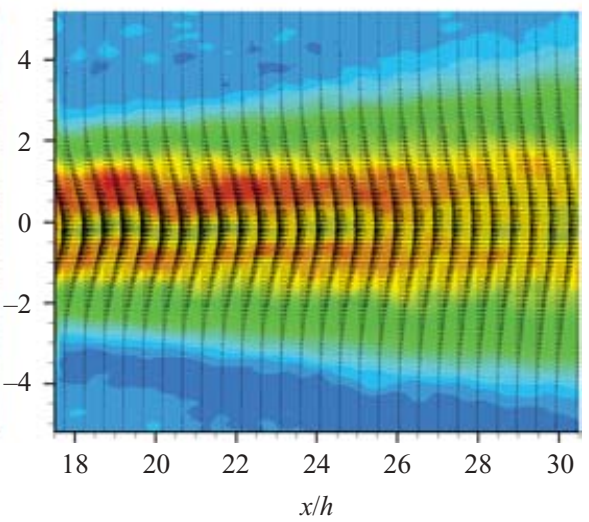

FIGURE 12. Contours of the normalized axial turbulence intensity in the post-injection region of the rectangular jet. $(a)$ Normal jet; $(b)$ with water injection.

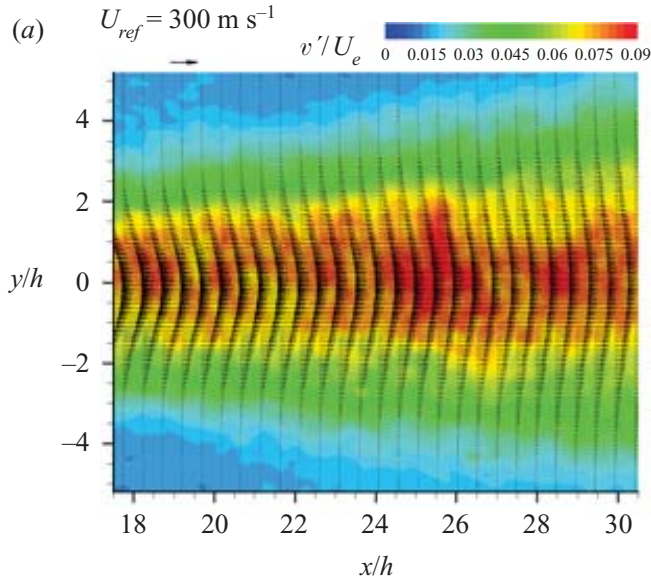

(b)

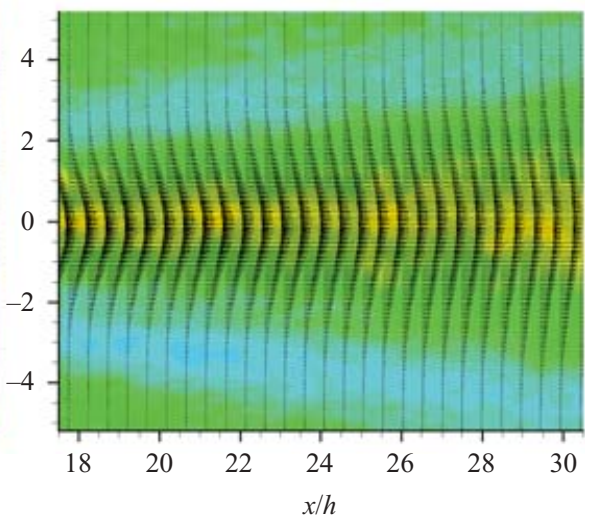

FIGURE 13. Contours of the normalized transverse turbulence intensity in the post injection region of the rectangular jet. (a) Normal jet; $(b)$ with water injection.

at all frequencies. These limited measurements are taken only to see relative changes in the spectra brought about by water injection. They are not meant to provide the far-field noise spectrum of the jet. However, direct absolute comparisons can be made from the far-field measurements made under anechoic conditions as discussed below.

The peak frequency of the spectra is observed at a much higher frequency than usually seen in the far-field spectra (figures 20 and 21). Debiasi \& Papamoschou (2001) have observed that the high-frequency components of the near-field spectra are significantly attenuated in the far field. The spectra shown in figure 17 show an unusually enhanced peak at about $35 \mathrm{kHz}$. By conducting tests in a separate rig, we have found that this peak comes from leaving the microphone protective grid on. The results from the same tests show that the frequency response of the microphone is unaffected by the protective grid for frequencies less than about $25 \mathrm{kHz}$. The observed reductions are an accurate representation of the possible levels of jet noise reduction due to the use of water injection. Reductions in the OASPL of about 
(a)

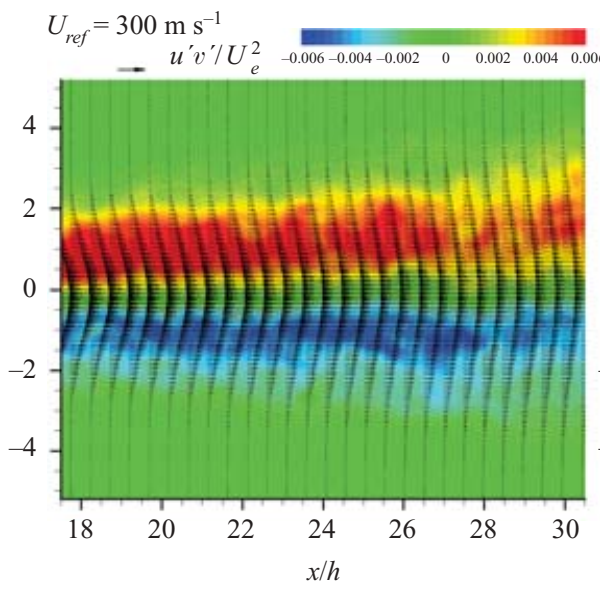

(b)

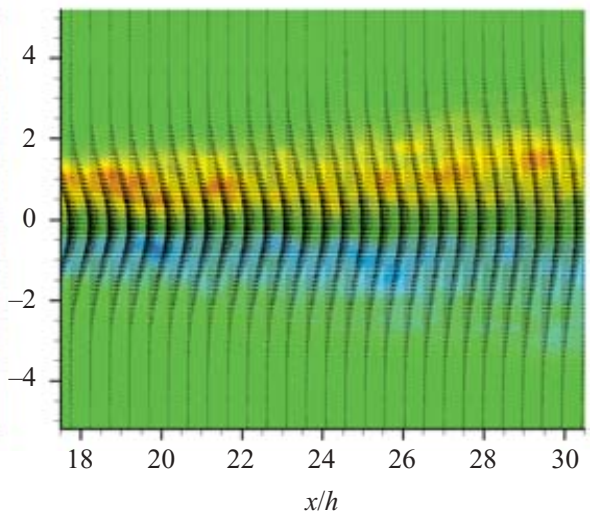

Figure 14. Contours of the normalized turbulent shear stress in the post-injection region of the rectangular jet. $(a)$ Normal jet; $(b)$ with water injection.
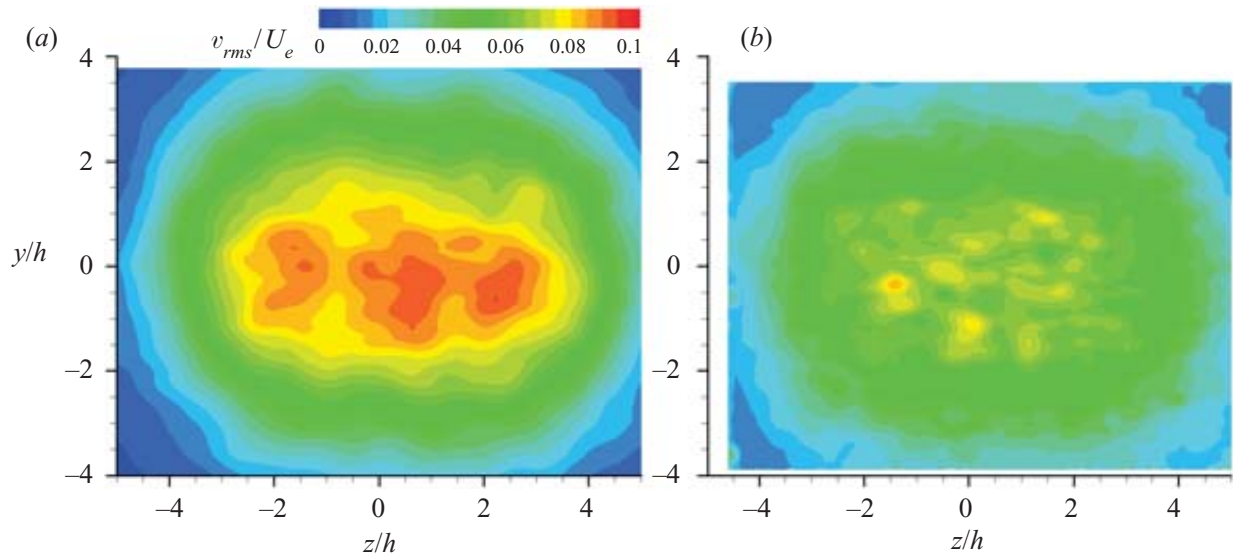

FIGURE 15. Contours of the cross-plane normalized transverse turbulence intensity distribution of the rectangular jet at $x / h=25$. (a) Normal jet; $(b)$ with water injection.

$6 \mathrm{~dB}\left(\dot{m}_{\text {water }} / \dot{m}_{\text {air }} \approx 0.1\right)$ and $2 \mathrm{~dB}\left(\dot{m}_{\text {water }} / \dot{m}_{\text {air }} \approx 0.05\right)$ are found for the cases of water injection at the nozzle exit and at the end of the potential core, respectively. Guided by this observation, experiments at $M=0.9$ were conducted with water being injected at the nozzle exit. Selected far-field noise results of this experiment are described below.

\subsubsection{Axisymmetric-jet far-field noise measurements}

Figure 18 shows the far-field OASPL directivity of $M_{j}=0.9$ normal axisymmetric jet. Also included in the figure are the data from Tanna, Dean \& Burrin (1976). The measured directivities agree reasonably well, suggesting that the acoustic results from the new facility are consistent with those reported in the literature. The effect of water injection on the OASPL is shown in figure 19 for two different microjet pressures corresponding to $12 \%$ and $17 \%$ of the primary jet mass flux. Significant $(6 \mathrm{~dB})$ reduction in the OASPL is measured in the peak radiation angle along with modest reductions at $90^{\circ}$. Since, the contributions of the sources due to large-scale structures are dominant in the aft quadrant, it is suggested that observed reductions in the 

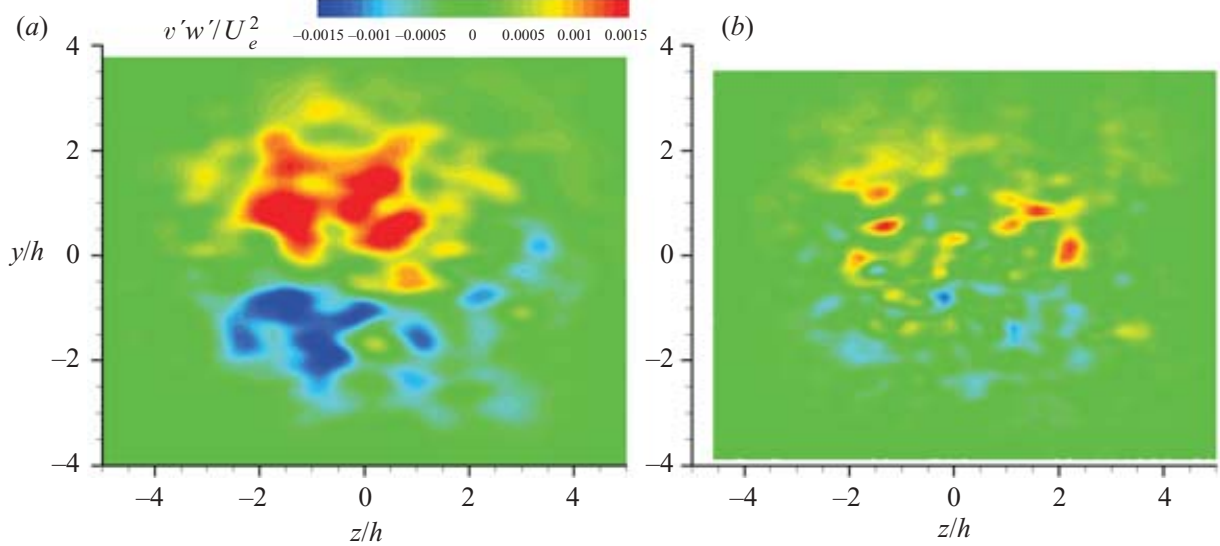

FiguRE 16. Contours of the cross-plane normalized shear stress distribution $\left(v^{\prime} w^{\prime}\right.$-component) of the rectangular jet at $x / h=25$. (a) Normal jet; $(b)$ with water injection.

downstream direction are primarily due to the suppression of the large-scale mixing noise. Based on the PIV results discussed above, it is believed that the turbulence reduction due to the break up of water droplets and the presence of a dispersed phase are responsible for noise suppression. The noise suppression at $90^{\circ}$ indicates that the small and intermediate scale turbulence noise sources are suppressed as well.

The effects of injection are further examined using the far-field narrowband spectra as shown in figure 20 and 21 for two different angles corresponding to the peak radiation angle and normal to the jet axis. The spectra show a broad peak at a non-dimensional frequency $\left(S t=f D / U_{j}\right)$ of about 0.2 at the peak radiation angle of $140^{\circ}$, which is consistent with the data in the literature (Krothapalli et al. 2003). The suppression at low frequencies without significant changes at high frequencies is clearly depicted in the figure. Other suppression techniques such as tabs and flexible filaments show increases at high frequencies without having any OASPL reductions, as shown by Simonich et al. (2001). In the present experiments, insignificant increase at high frequency are observed. Since low-frequency noise is known to consist mostly of contributions from the large-scale mixing, the water injection appears to interfere constructively, resulting in SPL reductions at low frequencies.

\section{Summary}

A series of experiments were performed to examine the influence of water injection on the flow and noise characteristics of an $M=1.44$ rectangular jet. A water spray consisting of $140 \mu \mathrm{m}$ drops (average drop size) was injected into the shear layer of the jet, covering a region of about $7 \mathrm{~h}$, at two different locations, at the nozzle exit and at the end of the potential core. The mass flow rate of the water was substantially lower $(\sim 5 \%-10 \%$ of the jet mass flow rate) than those used in earlier two-phase flow studies for similar applications. Phase Doppler particle analyser measurements indicate that drops break up quickly in the high-speed shear layer. At the end of the injection region, the drops are reduced to droplet clouds consisting mostly of $4 \mu \mathrm{m}$ size droplets.

Particle image velocity measurements in the post-injection region indicate that the droplet breakup process extending over an appreciable region results in significant turbulence attenuation. Mean velocity field in the post-injection region of the jet 

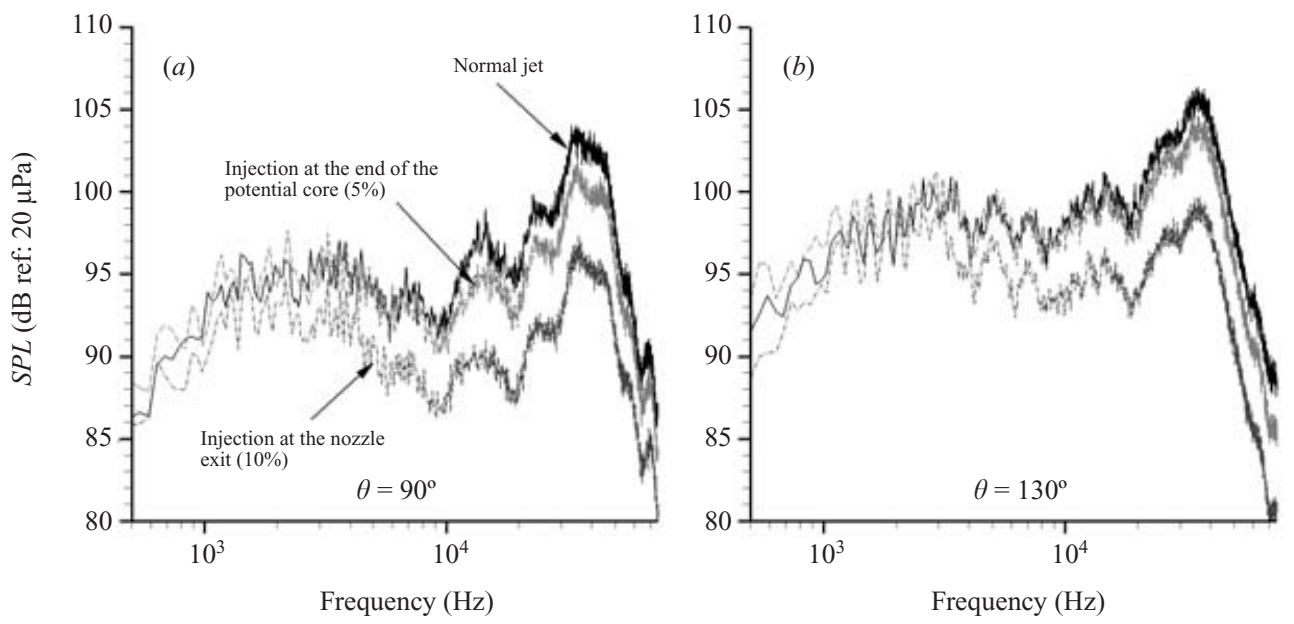

FiguRE 17. Near-field noise spectra of the rectangular jet at two different angles measured from the jet inlet axis $(a)$ Normal to the jet; $(b)$ peak radiation direction.

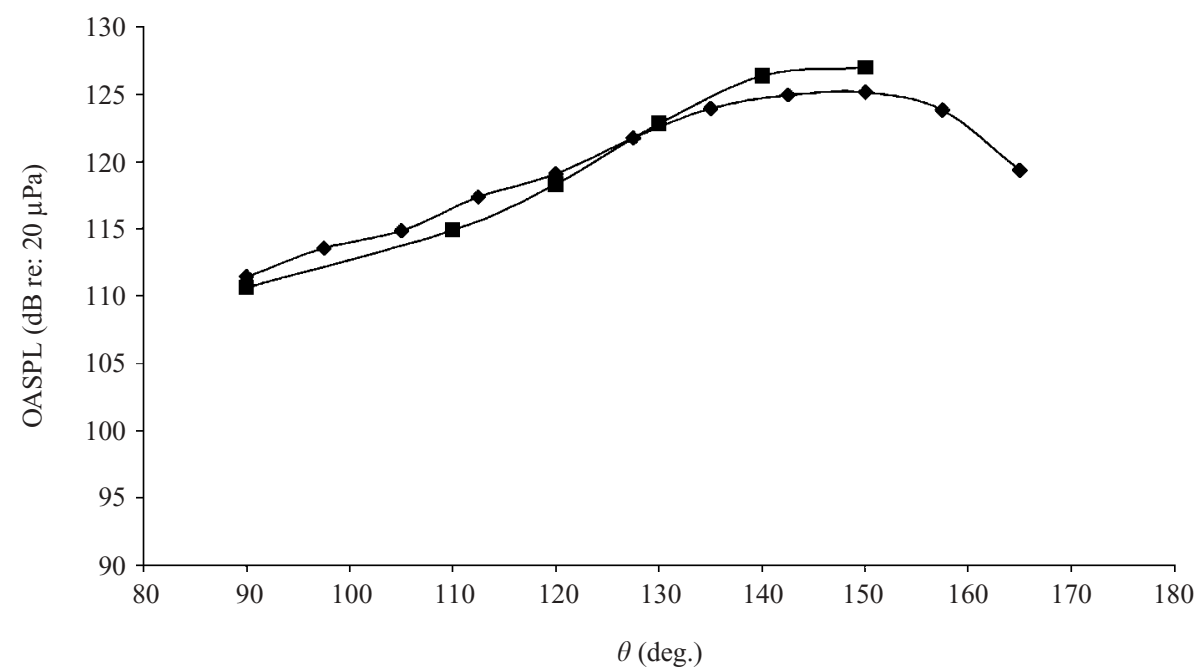

FIGURE 18. Far-field OASPL directivity of the axisymmetric jet at $r / D=64$. $\bullet$, Tanna et al. (1976) $\left(M=0.8, T_{o} / T_{a}=3.83, U_{j}=505 \mathrm{~m} \mathrm{~s}^{-1} ; \mathbf{\square}\right.$, Present results $\left(M=0.09, T_{o} / T_{a}=3.44, U_{j}=\right.$ $\left.525 \mathrm{~m} \mathrm{~s}^{-1}\right)$.

was found to be nearly identical with the normal jet to within the experimental uncertainty, suggesting that there was no apparent modification to the mean flow.

The narrowband spectral measurements of the far-field noise made on an axisymmetric $M=0.9$ heated jet indicate that water injection reduces the SPL at low frequencies without increasing the high-frequency noise. An OASPL reduction of about $6 \mathrm{~dB}$ was found at the peak radiation angle with $\dot{m}_{\text {water }}=0.17 \dot{m}_{j e t}$.

The results discussed in this paper clearly demonstrate the viability of water injection for noise suppression; however, several issues must be addressed. For example, the role of the parameters, such as the angle of injection, the mass flux ratio, the momentum flux ratio and the physical properties of the injection fluid on the noise suppression require further investigation. Since, DNS studies have advanced to 


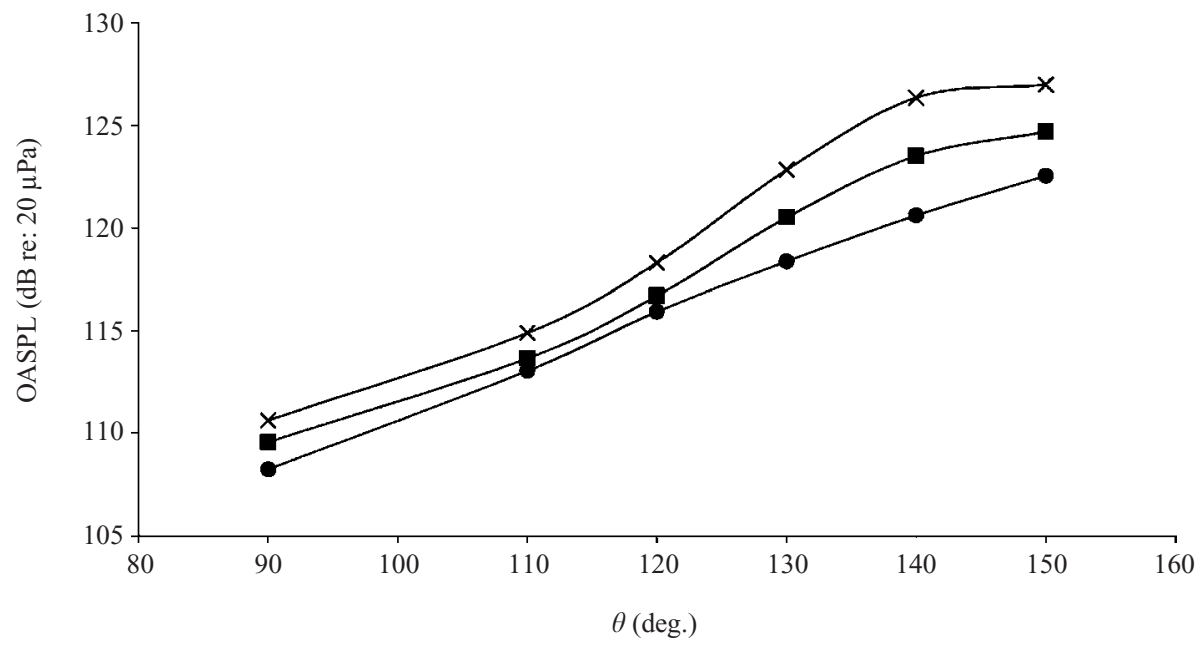

FIGURE 19. Effect of water injection on the far-field directivity of the axisymmetric jet. $\mathrm{X}$, normal jet; $\mathbf{\square}$, water injection at $2.07 \mathrm{Mpa} ; \bullet$, water injection at $3.45 \mathrm{Mpa}$.

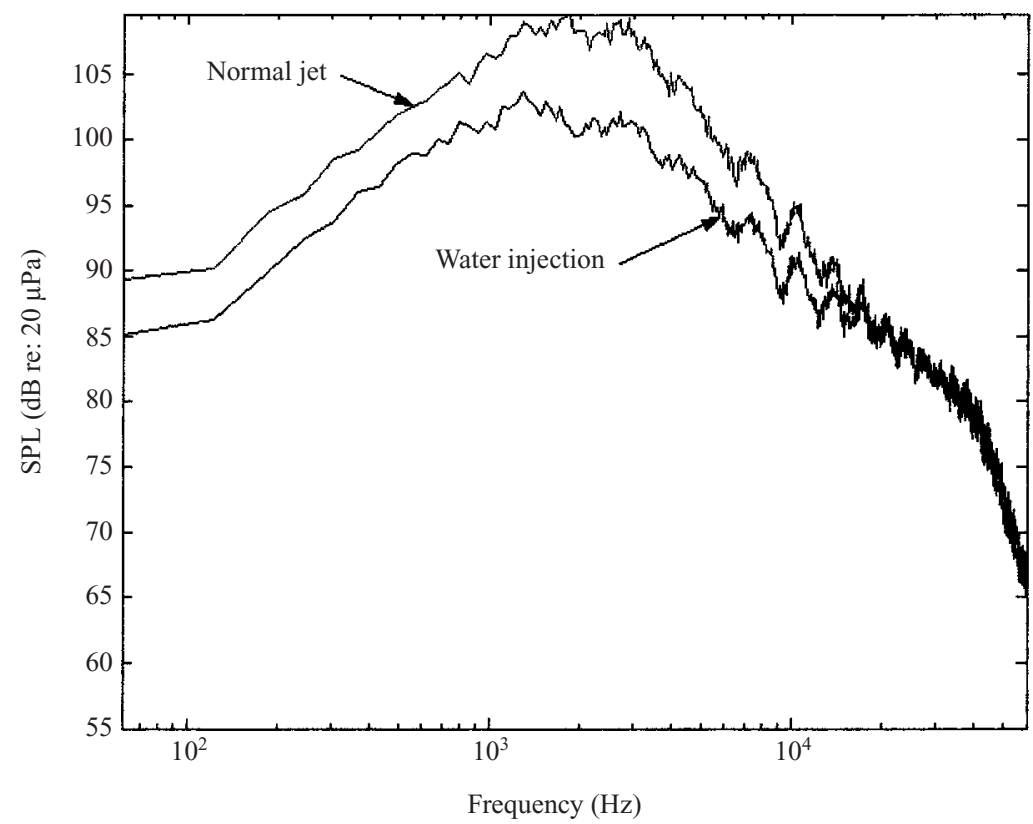

FIGURE 20. Narrowband far-field spectrum of the axisymmetric jet at $\theta=140^{\circ}$.

the extent of predicting radiated noise levels from both high subsonic and supersonic jets (Freund, Lele \& Moin 2000; Freund 2001); it would be of great interest to see if such computations can show significant noise suppression due to the use of water injection.

NASA Langley Research Center and Office of Naval Research supported the work described in this paper. Without the unwavering support and encouragement of $\mathrm{Dr}$ Dennis Bushnell this work would not have been carried out with vigour. Discussions 


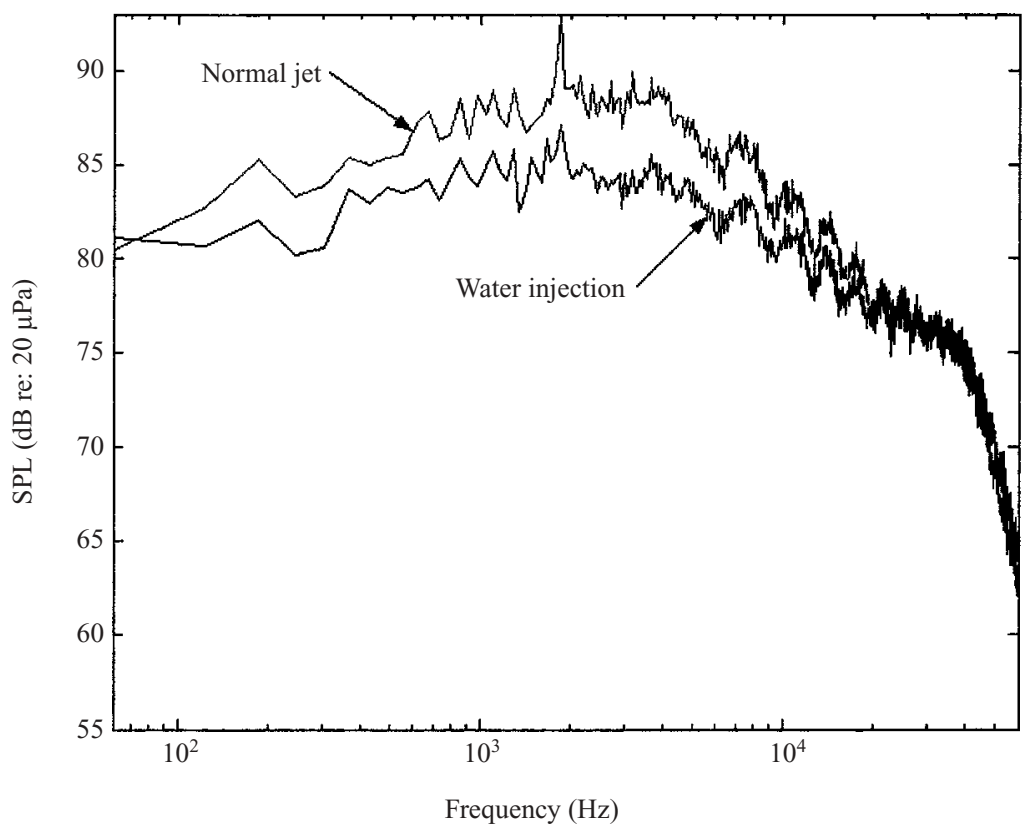

Figure 21. Narrowband far-field spectrum of the axisymmetric jet at $\theta=90^{\circ}$.

with Professor Vijay Arakeri have been useful. Dr Bahdir Alkislar's help in creating the figures is appreciated. We gratefully acknowledge the referees for helpful comments.

\section{Appendix. Water droplet characteristics}

A brief study was performed to obtain the relevant characteristics of drops and their evolution by the measurement of drop-size probability density functions using a TSI phase doppler particle analyser (PDPA/LDV) system Rev. 2.0 RSA. These measurements were used primarily to determine the droplet diameter at different locations in the jet. This study is not intended to provide a detailed account of the breakup mechanisms of liquid drops injected into a transverse high-velocity air stream (see Chou, Hsiang \& Faeth 1997; Liu \& Reitz 1997 and the references therein, on this subject). However, the resulting droplet characteristics are important here to ensure that the PIV velocity measurements accurately represent the flow physics described earlier.

The PDPA instrument used in this study was capable of measuring drop diameters from $0.5 \mu \mathrm{m}$ to $170 \mu \mathrm{m}$. The primary jet was not seeded during PDPA measurements. Figure 22 shows the probability density function of droplet diameter at the injector exit. The arithmetic mean diameter $\left(D^{10}\right)$ is $140 \mu \mathrm{m}$. The velocity of the drops at the injector exit was $20 \mathrm{~m} \mathrm{~s}^{-1}$ and decreases to about $10 \mathrm{~m} \mathrm{~s}^{-1}$ at the edge of the shear layer. From the PIV measurements, the magnitude of the free jet entrainment velocity was also found to be about $10 \mathrm{~m} \mathrm{~s}^{-1}$, suggesting that the drops enter the shear layer at the same velocity as the entrainment velocity.

The relative velocity between the drop and the gas varies from almost zero at the edge of the shear layer to about $400 \mathrm{~m} \mathrm{~s}^{-1}$ on the jet axis. In the region of interest, most of the droplets are found in the high-speed portion of the shear layer. Hence, for the purposes of calculating non-dimensional quantities, the relative velocity of the 


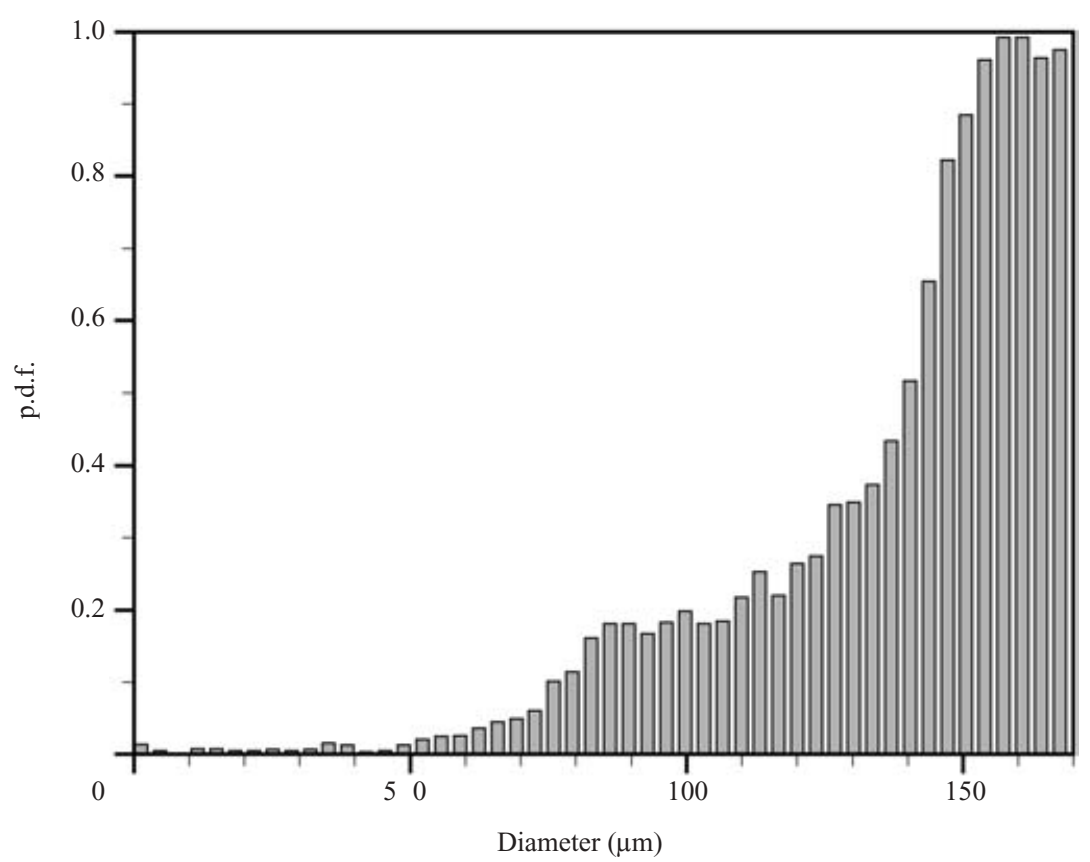

FIGURE 22. PDF of water droplets at the injector exit.

droplet is taken as $250 \mathrm{~m} \mathrm{~s}^{-1}$. From this and other information, we can then calculate the non-dimensional quantities of interest as follows.

Weber number of the drop:

$$
W e=\frac{\rho_{G} d_{0} u_{0}^{2}}{\sigma}=142,
$$

where $d_{0}, \sigma$ and $u_{0}$ are the initial drop diameter, surface tension of the water and its relative velocity to the flow, respectively. $\rho_{G}$ is the density of air. The subscripts $L$ and $G$ denote liquid and gas properties.

Reynolds number of the drop:

$$
R e=\frac{\rho_{G} u_{0} d_{0}}{\mu_{G}}=2250 .
$$

Ohnesorge number:

$$
O h=\frac{\mu_{L}}{\sqrt{\rho_{0} d_{0} \sigma}}=9.5 \times 10^{-3}
$$

From the breakup regime map of Hsiang \& Faeth (1995) when based on the Weber and Ohnesorge numbers calculated above, it is seen that the breakup process of the drops in the present study is of the shear type $\left(10^{2}<W e<10^{3}, 10^{-4}<O h<10^{-1}\right)$.

The temporal properties of the drop breakup in the shear breakup regime are estimated here based on the analysis of Chou et al. (1997). One of the important parameter is the characteristic breakup time, $t^{*}$ defined as follows (Ranger \& Nicholls 1969):

$$
t^{*}=\frac{d_{0} \sqrt{\left(\rho_{L} / \rho_{G}\right)}}{u_{0}} .
$$




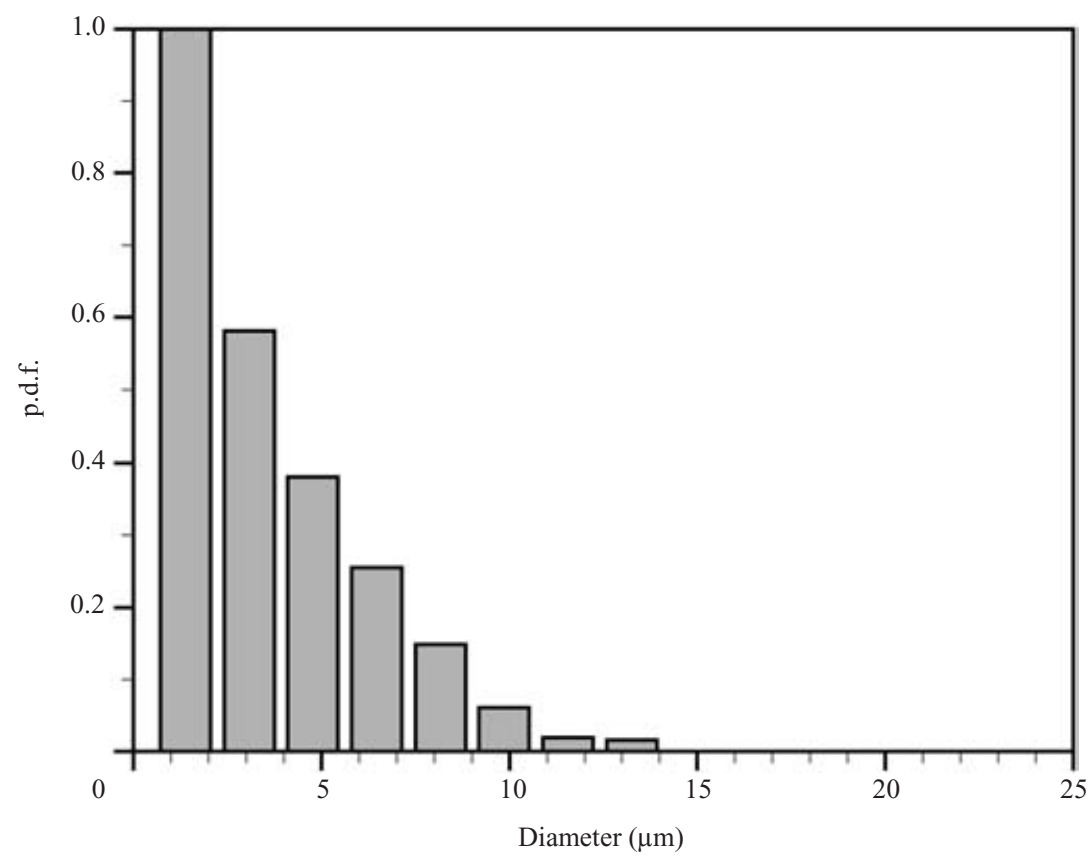

FIGURE 23. Typical droplet size distribution measured at $x / h=26$ and $y / h=1.5$.

Liang, Easter \& Gharakhari (1988) show that the onset and end of breakup (where the liquid removal from the parent drop begins and ends) occur at $t / t^{*}=1.5$ and 5.5 , respectively. In the present study, $t^{*}=1.5 \times 10^{-5} \mathrm{~s}$. This implies that for a droplet injected at $x / h=10$; breakup begins at $x / h=10.5$ and ends at $x / h=12.5$, using an average velocity of the drop of $250 \mathrm{~m} \mathrm{~s}^{-1}$. Chou et al. (1997) have proposed a model for shear breakup in which they find the temporal variation of the Sauter mean diameter (SMD) $\left(D^{32}=\right.$ diameter of a droplet whose ratio of volume to surface area is equal to that of the complete spray sample). They find two regimes of breakup: a transient process which occurs from initiation of breakup until a time $t_{c}\left(t_{c} / t^{*}=0.002\left(\rho_{G} / \rho_{L}\right)^{1 / 2} u_{0} d_{0} / v_{L}\right)$ and a quasi-steady process from $t_{c}$ to $5.5 t^{*}$ where the variation of the SMD becomes almost a constant $\left(S M D(t) / d_{0}=0.09\right)$. In the present application, an estimation of these quantities yield, $t_{c}=34.7 \mu$ s and the largest possible droplet diameter $=12 \mu \mathrm{m}$. Our PDPA measurements indeed show that there are such large particles, but they are present only less than $2.5 \%$ of the time (figure 23 ). However, the above model does not contain any information about the p.d.f. distribution of the final droplet size. Martinez-Bazan, Montanes \& Lasheras (1999a, $b$ ) have recently proposed a model for the breakup and the p.d.f. (probability density function) of air bubbles in a water jet. For very dilute systems (negligibly small rates of collision between particles), and in the absence of evaporation effects, the model can predict the bubble size p.d.f. of the 'daughter' bubbles resulting from the shattering or breakup of larger mother bubble immersed into a fully developed turbulent water flow. Although, this model can be modified to the current situation to yield the final droplet size p.d.f., it is beyond the scope of the present work to carry it out. Instead, we determine the final droplet size p.d.f. from the PDPA measurements.

Figure 23 shows a typical droplet size p.d.f. measured at $x / h=26$ and $y / h=1.5$. The data indicate that very few droplets having sizes greater than about $5 \mu \mathrm{m}$ now 


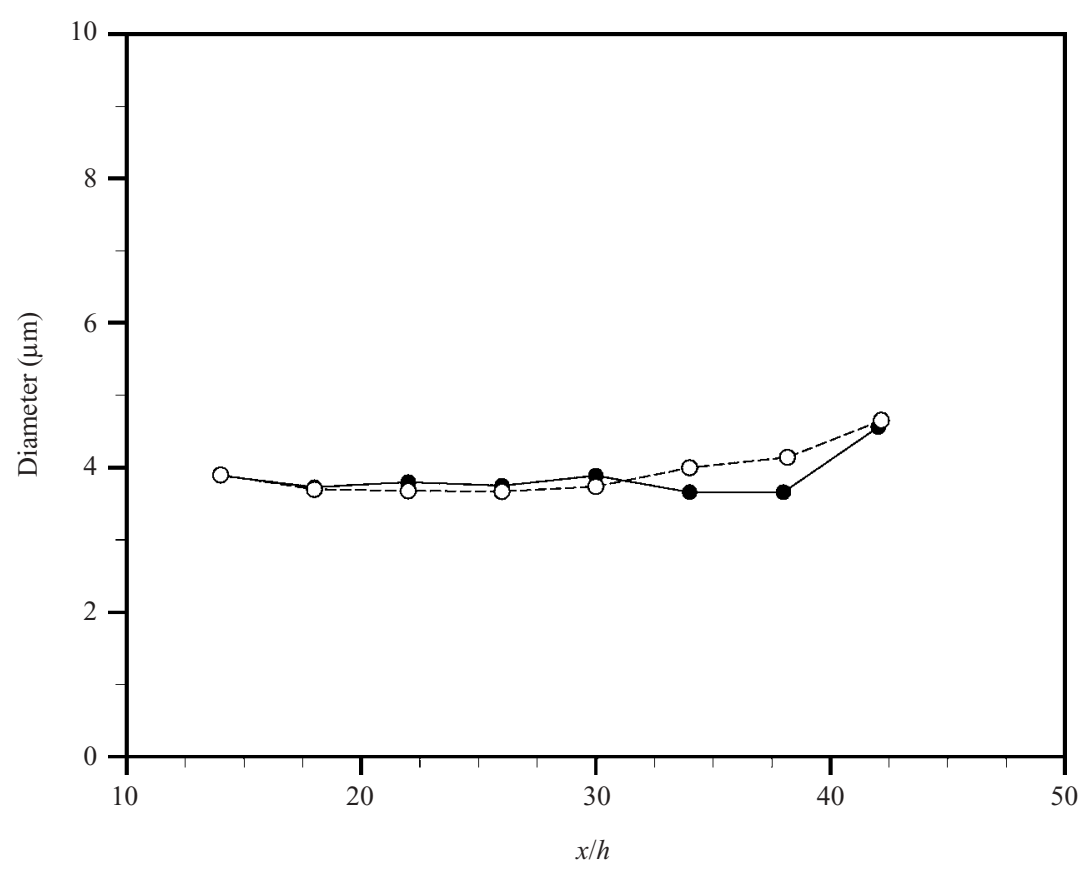

FIGURE 24. Droplet diameter variation with downstream distance in the shear layer at $y / h=1.5$. @, upper shear layer; $\bigcirc$, lower shear layer.

exist in the shear layer. As expected from the discussion above, the drops break up quickly from an initial size of $140 \mu \mathrm{m}$ at the injector exit to the size of a few micrometres. It is expected that the larger left-over droplets will be dispersed further due to turbulence; the estimated size of the resulting droplets being about $4 \mu \mathrm{m}$ (Martinez-Bazan et al. 1999). The variation of the arithmetic mean droplet diameter along the maximum shear line in the shear layer is shown in figure 24. With increasing downstream distance, mean droplet diameter remains constant suggesting that the drop breakup process is mostly complete within the injection region and no more breakup takes place in the region where the PIV measurements are made. However, to ensure that no larger droplets were present in the region of the measurement, droplet size p.d.f.s were obtained across the jet at several downstream locations. A typical transverse profile of the arithmetic mean droplet diameter is shown in figure 25 . The data clearly show that the droplet size remains uniform across the jet. These observations are consistent with those of Martinez-Bazan et al. (1999a, b).

The effect of the inertia of a particle on its flow-tracking accuracy in a compressible turbulent shear layer was discussed in detail by Samimy \& Lele (1991). They have shown that the particle response is well characterized by a parameter $\tau$, the ratio of particle response time to the flow time scale, defined as

$$
\tau=\frac{\tau_{p}}{\tau_{f}},
$$

where $\tau_{f}=10 \delta_{u o} /\left(U_{1}-U_{2}\right), \delta_{u o}$ is the initial vorticity thickness and $\tau_{p}=\rho_{p} d_{p}^{2} / 18 \mu$.

For the conditions of the present experiment, the parameter $\tau$, was estimated to be about 0.2 . The measured arithmetic mean droplet diameter of $4 \mu \mathrm{m}$ was used in this estimation. Samimy \& Lele (1991) show that the velocity measurement errors grow linearly with $\tau$ with approximately $2 \%$ error for $\tau=0.2$. Based on this observation, 


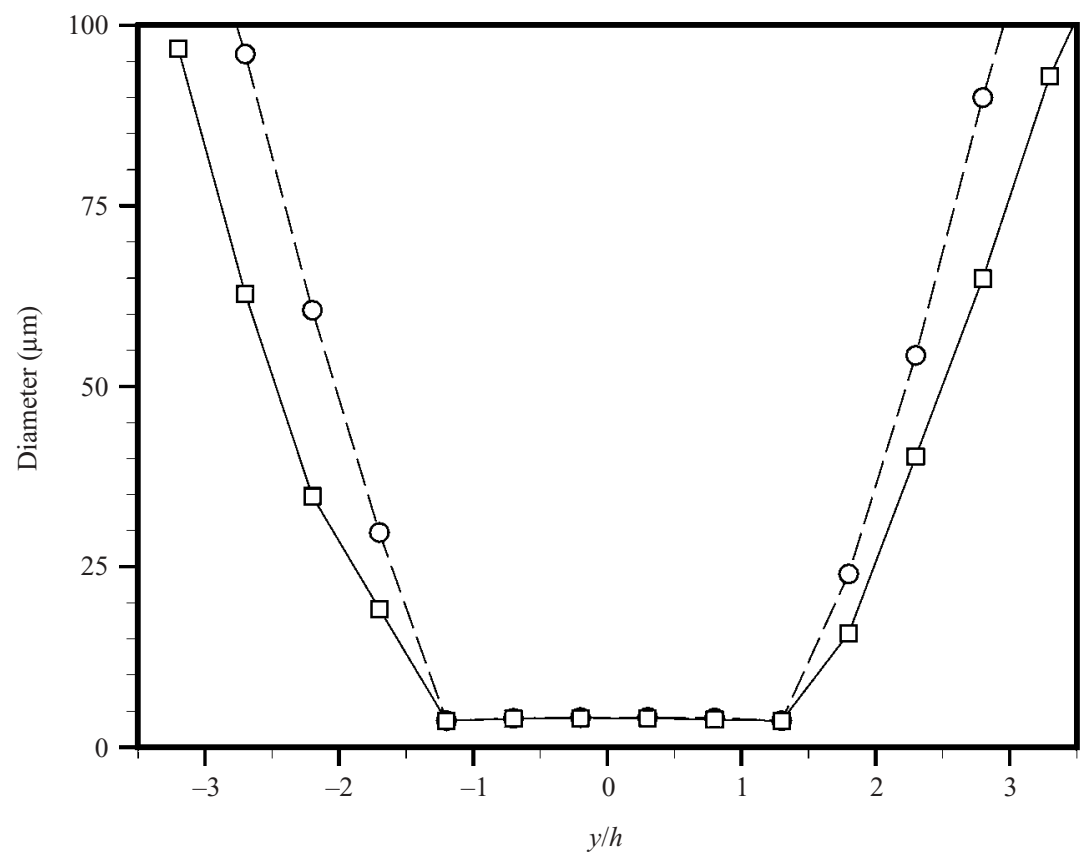

FiguRE 25. Droplet size variation across the jet in the central $(x, y)$-plane. $\bigcirc, x / h=30 ; \square, 40$.

and since both seeding particle and water droplet sizes are within the acceptable range, the PIV velocity measurements are expected to be within an uncertainty associated with an experiment of this nature, typically about $1 \% \sim 2 \%$.

To further minimize the effect of larger water droplet size on PIV processing, the images of particles of intensity larger than a threshold value (determined from the average intensity of the normal jet images) were removed using a filter. As a result, most of the contribution to the image cross-correlation remains that of the seed particles.

\section{REFERENCES}

Alkislar, M. B. 2001 Flow characteristics of a screeching rectangular jet: a stereoscopic PIV study. $\mathrm{PhD}$ thesis, Florida State University.

Alkislar, M. B., Krothapalli, A. \& Lourenco, L. M. 2003 Structure of a screeching rectangular jet: a stereoscopic PIV study. J. Fluid Mech. 489, 121-154.

Alvi, F. S., Krothapalli, A., Washington, D. \& King, C. J. 1996 Aeroacoustic properties of a supersonic diamond-shaped jet. AIAA J. 34, 1562-1569.

Bishop, K. A., Ffowcs Williams, J. F. \& Smith, W. 1971 On the noise of the unsuppressed high-speed jet. J. Fluid Mech. 50, 21-31.

Blackstock, D. T. 2000 Fundamentals of Physical Acoustics. John Wiley.

Chou, W. H., Hsiang, L. P. \& Faeth, G. M. 1997 Temporal properties of drop breakup in the shear breakup regime. Intl J. Multiphase Flow 23, 651-669.

Crighton, D. G. 1977 Orderly structure as a source of jet exhaust noise: survey lecture. In Structure and Mechanisms of Turbulence II (ed. H. Fiedler), Lecture Notes in Physics, vol. 76, pp. 154-170. Springer.

Debiasi, M. \& Papamoschou, D. 2001 Noise from imperfectly expanded supersonic coaxial jets. AIAA J. 39, 388-395.

Ffowcs Williams, J. E. 1965 On the development of Mach waves radiated by small disturbances. J. Fluid Mech. 22, 49-55. 
Ffowcs Williams, J. E. \& MaidaniK, G. 1965 The Mach wave field radiated by supersonic turbulent shear flows. J. Fluid Mech. 21, 641-657.

Ffowcs Williams, J. E., Simson, J. \& Virchis, V. J. 1975 Crackle: an annoying component of jet noise. J. Fluid Mech. 71, 251-271.

FreUnd, J. B. 2001 Noise sources in a low-Reynolds-number turbulent jet at Mach 0.9. J. Fluid Mech. 438, 277-305.

Freund, J. B., Lele, S. K. \& Moin, P. 2000 Numerical simulation of a Mach 1.92 turbulent jet and its sound field. AIAA J. 38, 2023-2031.

Hoch, R. \& Hawkins, R. 1974 Recent studies into Concorde noise reduction. AGARD CP 131 on Noise Mechanisms, pp. 19-1-19-14.

Hsiang, L. P. \& FaEth, G. M. 1995 Drop deformation and breakup due to shock wave and steady disturbances. Intl J. Multiphase Flow 21, 545-560.

Knudsen, V. O., Wilson, J. V. \& Anderson, N. S. 1948 The attenuation of sound in fog and smoke. J. Acoust. Soc. Am. 20, 849-857.

Krothapalli, A., Arakeri, V. \& Greska, B. 2003 Mach wave radiation: a review and an extension. AIAA Paper 2003-1200.

Krothapalli, A., Baganoff, D. \& Karamcheti, K. 1981 On the mixing of a rectangular jet. J. Fluid Mech. 107, 201-220.

Krothapalli, A., Hsia, Y., Baganoff, D. \& Karamcheti, K. 1986 The role of screech tones in mixing of an underexpanded rectangular jet. 106, J. Sound Vib. 106, 119-143.

Krothapalli, A., Venkatakrishnan, L., Elavarasan, R. \& Lourenco, L. $2000 a$ Supersonic jet noise suppression by water injection. AIAA Paper 2000-2025.

Krothapalli, A., Venkatakrishnan, L. \& Lourenco, L. $2000 b$ Crackle: a dominant component of supersonic jet mixing noise. AIAA Paper 2000-2024.

Kulick, J. D., Fessler, J. R. \& Eaton, J. K. 1994 Particle response and turbulence modification in fully developed channel flow. J. Fluid Mech. 277, 109-134.

LAU, J. C. 1980 Laser velocimeter correlation measurements in subsonic and supersonic jets. J. Sound Vib. 70, 85-101.

LaU, J. C., Morris, P. J. \& Fisher, M. 1979 Measurements in subsonic and supersonic free jets using a laser velocimeter. J. Fluid Mech. 93, 1-27.

Laufer, J., Schlinker, R. \& KaPlan, R. E. 1976 Experiments on supersonic jet noise. AIAA J. 14, 489-504.

Laufer, J., Kaplan, R. E. \& Chu, W. T. 1973 Acoustic modeling of the jet noise abatement problem. Proc. Interagency Symp. in Transportation Noise, Stanford University, Stanford, CA.

Liang, P. Y., Eastes, T. W. \& Gharakhari, A. 1988 Computer simulations of drop deformation and drop breakup. AIAA Paper 88-3142.

LiU, Z. \& ReITZ, R. D. 1997 An analysis of the distortion and breakup mechanisms of high speed liquid drops. Intl J. Multiphase Flow 23, 631-650.

Lourenco, L. M. \& Krothapalli, A. 2000 TRUE resolution PIV: a mesh-free second order accurate algorithm. Proc. Intl Conf. Application of Lasers to Fluid Mech. Lisbon, Portugal.

Marble, F. E. \& Candel, S. M. 1975 Acoustic attenuation in fans and ducts by vaporization of liquid droplets. AIAA J. 13, 634-639.

Marble, F. E. \& Wooten, D. C. 1970 Sound attenuation in a condensing vapor. Phys. Fluids 13, 2657-2664.

Martinez-Bazan, C., Montanes, J. L. \& Lasheras, J. C. 1999 On the breakup of an air bubble injected into fully turbulent flow. Part 1. Breakup frequency. J. Fluid Mech. 401, $157-182$.

Martinez-Bazan, C., Montanes, J. L. \& Lasheras, J. C. 1999 On the breakup of an air bubble injected into fully turbulent flow. Part 2. Size PDF of the resulting daughter bubbles. J. Fluid Mech. 401, 183-207.

MashaYeK, F. 1998 Droplet-turbulence interactions in low-Mach-number homogeneous shear twophase flows. J. Fluid Mech. 367, 163-203.

Oertel, H. 1979 Mach wave radiation of hot supersonic jets. Mechanics of Sound Generation in Flows (ed. E. A. Müller), pp. 275-281. Springer.

Oertel, H., Gatau, F. \& George, A. 1981 Dynamik der Machwellen in der Umgebung von Überschallstrahlen. ISL Rep. R 124/81, Institut franco-allemand de St Louis, Saint-Louis, France. 
Oertel, H \& Patz, G. 1981 Wirkung von Unterschallmanteln auf die Machwellen in der Umgebung von Überschallstrahlen. ISL Rep. RT 505/81, Institut franco-allemand de St Louis, SaintLouis, France.

Papamoschou, D. 1997 Mach wave elimination from supersonic jets. AIAA J. 35, 1604-1611.

Phillips, O. M. 1960 On the generation of sound by supersonic turbulent shear layers. J. Fluid Mech. 9, 1-28.

Ranger, A. A. \& Nichols, J. A. 1969 The aerodynamic shattering of liquid drops. AIAA J. 7, $285-290$.

Samimy, M. \& Lele, S. K. 1991 Motion of particles with inertia in a compressible free shear layer. Phys. Fluids A 3, 1915-1923.

Seiner, J. M., Ponton, M. K., Jansen, B. J. \& Lagen, N. T. 1992 The effects of temperature on supersonic jet noise emission. DGLR/AIAA Paper 92-02-045.

Simonich, J. C., Narayanan, S., Barber, T. J. \& Nishimura, M. 2001 Aeroacoustic characterization, noise reduction and dimensional scaling effects of high subsonic jets. AIAA J. 39, 2062-2069.

TAM, C. K. W. 1998 Jet noise: since 1952. Theo. Comput. Fluid Dyn. 10, 393-406.

TANNA, H. K., DeAn, P. D. \& BuRrin, R. H. 1976 The generation and radiation of supersonic jet noise. AFAPL-TR 76-65-vol. III, Air Force Aero-Propulsion Laboratory, Wright-Patterson Air Force Base.

Zoppellari, E. \& Juve, D. 1997 Reduction of jet noise by water injection. AIAA Paper 97-1622-CP, pp. 266-274. 